The Astrophysical Journal, 659:314-330, 2007 April 10

(C) 2007. The American Astronomical Society. All rights reserved. Printed in U.S.A.

\title{
THE RADIO SPECTRA OF THE COMPACT SOURCES IN ARP 220: A MIXED POPULATION OF SUPERNOVAE AND SUPERNOVA REMNANTS
}

\author{
Rodrigo Parra and John E. Conway \\ Onsala Space Observatory, Onsala, Sweden \\ Philip J. Diamond and Hannah Thrall \\ Jodrell Bank Observatory, Macclesfield, UK \\ Colin J. Lonsdale \\ MIT Haystack Observatory, Westford, MA \\ Carol J. Lonsdale \\ Infrared Processing and Analysis Center, California Institute of Technology Pasadena, CA \\ AND \\ HARDing E. SMITH \\ Center for Astrophysics and Space Sciences, University of California, San Diego, La Jolla, CA \\ Received 2006 September 22; accepted 2006 December 2
}

\begin{abstract}
We report the first detection at multiple radio wavelengths $(13,6$, and $3.6 \mathrm{~cm})$ of the compact sources within both nuclei of the ultraluminous infrared galaxy Arp 220, presenting radio spectra of the 18 detected sources. In just over half of these, we find that these spectra and other properties are consistent with the standard model of powerful Type IIn supernovae interacting with their preexplosion stellar wind. The rate of appearance of new radio sources identified with these supernova events suggests that an unusually large fraction of core-collapse supernovae in Arp 220 are highly luminous, possibly implying a radically different stellar initial mass function or stellar evolution compared to galactic disks. Another possible explanation invokes very short $\left(\sim 3 \times 10^{5} \mathrm{yr}\right)$ intense $\left(\sim 10^{3} M_{\odot} \mathrm{yr}^{-1}\right)$ star formation episodes with a duty cycle of $\sim 10 \%$. A second group of our detected sources, consisting of the brightest and longest monitored sources at $18 \mathrm{~cm}$, do not easily fit the radio supernova model. These sources show a range of spectral indexes from -0.2 to -1.9 . We propose that these are young supernova remnants that have just begun interacting with a surrounding ISM with a density between $10^{4}$ and $10^{5} \mathrm{~cm}^{-3}$. One source is probably resolved at $3.6 \mathrm{~cm}$ wavelength with a diameter $0.9 \mathrm{pc}$. In the western nucleus we estimate that the ionized component of the ISM gives rise to foreground free-free absorption with opacity at $18 \mathrm{~cm}$ of $<0.6$ along the majority of lines of sight. Other sources may be affected by absorption with opacity in the range $1-2$. These values are consistent with previous models as fitted to the radio recombination lines and the continuum spectrum.
\end{abstract}

Subject headings: galaxies: individual (Arp 220) — galaxies: starburst

\section{INTRODUCTION}

Studies of radio supernovae (RSNe) and supernova remnants (SNRs) in starburst galaxies can provide unique information about stellar evolution and interstellar medium (ISM) properties in regions completely hidden by dust at optical/IR wavelengths. Theoretical models of the radiation emitted by the interaction of the supernova (SN) blast wave with the circumstellar medium (CSM) or the local ISM (Chevalier 1982a, 1982b; Chevalier \& Fransson 1994, 2001; Weiler et al. 2002) combined with radio multiwavelength/multiepoch studies of such RSNe/SNRs (McDonald et al. 2001; Neff et al. 2004; Alberdi et al. 2006; Bartel et al. 2002) can be used to trace the physical conditions of the gas swept up as the $\mathrm{SN}$ evolves. In addition, from the rate of newly observed RSNe estimates can be made of the death rate of massive stars. Adopting a stellar initial mass function (IMF) and a burst lifetime longer than that of SNe progenitors, this can be converted into a star formation rate (SFR) and compared to other estimates. This intercomparison can set constrains on stellar population properties such as the shape of the stellar IMF.

For over a decade, the ULIRG Arp 220 has been the object of a high-sensitivity VLBI campaign at a wavelength of $18 \mathrm{~cm}$ to observe the evolution of a cluster of unresolved radio sources initially identified as Type IIn RSNe (Smith et al. 1998; Lonsdale et al. 2006). In this paper we present new observations in which we detect these sources for the first time at wavelengths shorter than $18 \mathrm{~cm}$ (i.e., at 13, 6, and $3.6 \mathrm{~cm}$ ) at mJy levels. Previous attempts to observe these sources at a wavelength of $6 \mathrm{~cm}$ (Rovilos et al. 2005) reported nothing above a $5 \sigma$ level of $250 \mu \mathrm{Jy}$. It seems that these earlier observations failed because a too distant phase calibrator was used (see $\S 2.4$ ). Now that we have made shortwavelength detections we can for the first time study the source spectra and check for consistency with SN/SNR models, in particular checking whether the extreme starburst environment of Arp 220 modifies their properties compared to SNe/SNRs in galactic disks.

The plan of the paper is as follows. In $\S 2$ we discuss the new observations and the reduction of the data. Section 3 presents our primary observational results, including the source spectra, structures, and the spatial distribution of the compact sources. In $\S 4$ we discuss the source spectra in more detail and fit simple synchrotron plus free-free absorption (FFA) models to these spectra. In $\S 5$ in light of new and published information we discuss physical models for the compact sources, comparing in particular to the expectations of SN and SNR models. We also discuss in detail the various subclasses of sources we have identified, 
TABLE 1

VLBI OBSERVATIONS of Arp 220

\begin{tabular}{|c|c|c|c|c|c|}
\hline Epoch & Code & $\begin{array}{c}\lambda \\
(\mathrm{cm})\end{array}$ & Array & $\begin{array}{c}\sigma_{\lambda} \\
\left(\mu \mathrm{Jy} \text { beam }^{-1}\right)\end{array}$ & $\begin{array}{c}\text { Beam Size } \\
\left(\mathrm{mas}^{2}\right)\end{array}$ \\
\hline $2003.01 \ldots$. & BN022 & 6.02 & VLBA & 147.01 & $4.4 \times 2.0$ \\
\hline $2003.85 \ldots \ldots$ & GD $17 A^{a}$ & 18.18 & GVLBI & 9.00 & $5.9 \times 2.7$ \\
\hline $2005.16 \ldots$. & ЕР049 & 6.02 & $\mathrm{~Eb}, \mathrm{~Wb}, \mathrm{Ar}$ & 42.08 & $200 \times 200^{c}$ \\
\hline $2005.18 \ldots \ldots$ & GD17B ${ }^{b}$ & 18.18 & GVLBI & 9.00 & $7.6 \times 2.9$ \\
\hline \multirow[t]{3}{*}{$2006.02 \ldots \ldots$} & BP129 & 13.26 & VLBA & 129.54 & $6.6 \times 3.6$ \\
\hline & & 6.02 & & 86.02 & $3.3 \times 1.8$ \\
\hline & & 3.56 & & 86.73 & $3.1 \times 1.7$ \\
\hline
\end{tabular}

${ }^{\text {a }}$ Lonsdale et al. (2006).

b H. Thrall et al. 2007, in preparation.

c Sensitive only to $<1$ mas features. See text for details.

set limits on the properties of the ionized ISM and speculate about their implications for stellar evolution and the SFR. Finally, in $\S 6$ we summarize our conclusions and discuss future prospects.

\section{OBSERVATIONS AND DATA REDUCTION}

\section{1. $6 \mathrm{~cm}$ Global VLBI}

Two of us (R. P. and J. C.) observed Arp 220 at $6 \mathrm{~cm}$ on 2005 February 27 with a short snapshot VLBI observation as part of the European VLBI network (EVN) project EP049, which is a survey of a large sample of infrared-selected galaxies known as COLA (Compact Objects in Low-Power AGNs; Corbett et al. 2002. 2003; Parra et al. 2005). These observations involved the Effelsberg (Eb), Westerbork (Wb), and Arecibo (Ar) Telescopes in one of the first user experiments scheduled at a data rate of 1 Gbit $\mathrm{s}^{-1}$. The full results of this survey will be given elsewhere (see R. Parra et al. 2007, in preparation). Like all other sources in the sample, Arp 220 was observed for only 10 minutes. Despite the short integration time, the combination of the high bit rate and very large telescopes resulted in an untapered noise level of only $\sigma=38 \mu \mathrm{Jy}$ on the Ar-Eb baseline.

The data were correlated on the EVN correlator at the Joint Institute for VLBI in Europe (JIVE) in the Netherlands. Classic AIPS was used for the calibration stage and fringe fitting, resulting in a detection with a signal-to-noise ratio of $\sim 60$. We then used our own software to make a delay-rate map (see Thompson et al. 2001 and references therein) from the Ar-Eb data, which showed two sources associated with the eastern and western nuclei with fluxes of 1.0 and $2.9 \mathrm{mJy}$, respectively (see Fig. 1). A delay-rate map made from the Ar-Wb data was similar but had larger noise. The significance of these maps is that, although they appear to have low resolution, they are in fact only sensitive to structures that are compact on the Ar-Eb baseline. ${ }^{1}$ Therefore, although for Figure 1 the delay-rate beam is large ( 200 mas), the map includes only emission contributed by features that are less then 2 mas in size and so does not include any of the extended emission seen in previous $6 \mathrm{~cm}$ MERLIN maps (Rovilos et al. 2003). This image therefore provided the first evidence for the detection of the Arp 220 compact features at $6 \mathrm{~cm}$. Given the large bandwidths now spanned by $1 \mathrm{Gbit} \mathrm{s}^{-1}$ observations, this delay-rate (or "single

\footnotetext{
1 Delay-rate map is a representation of the true brightness distribution after high-pass filtering and convolving with a delay-rate beam. Formally, it is the result of the following three steps: (1) First, the true image $I(x, y)$ multiplied it by $\exp \left[-2 \pi i\left(u_{0} x+v_{0} y\right)\right]$, where $u_{0}$ and $v_{0}$ are the $u v$ coordinates of the center of the patch of the $u v$ plane sampled by the time and frequency range of the single baseline data. (2) The result is then convolved with a large delay-rate beam set by the Fourier transform of the sampled $u v$ patch. (3) The amplitude of the resulting complex image is taken.
}

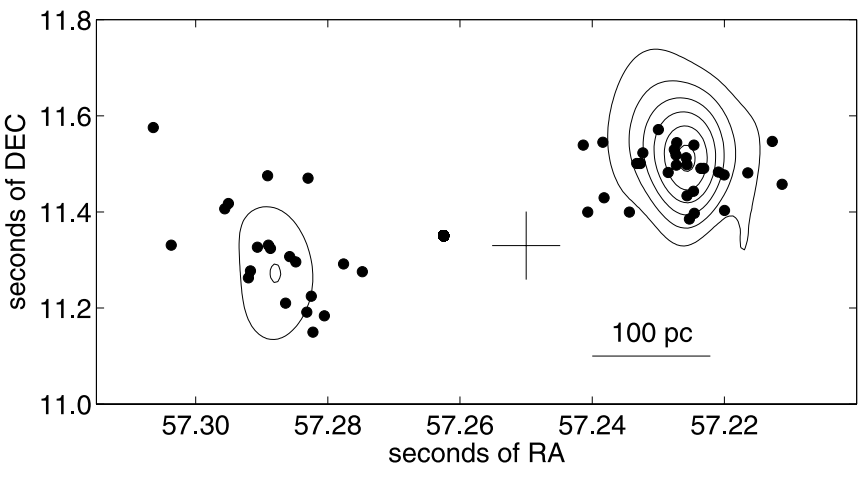

FIG. 1.-Overall view of the Arp 220 nuclear region. Filled circles are the corrected positions (see $\S 2.4$ for details) of the 49 compact sources at $18 \mathrm{~cm}$ cataloged by Lonsdale et al. (2006). Contours show a $6 \mathrm{~cm}$ delay-rate map ("single-baseline snapshot image") obtained from a 10 minute scan with the Ar-Eb baseline in project EP049. Contours are drawn $500 \mu \mathrm{Jy}_{\text {beam }^{-1}}$ apart starting from $500 \mu \mathrm{Jy} \mathrm{beam}^{-1}$. The data was tapered in time and frequency using a Chebyshev window to reduce sidelobes, resulting in a delay-rate beam of FWHM $\sim 200$ mas and a map noise of $\sim 42 \mu \mathrm{Jy}$ beam $^{-1}$. The cross indicates the reference position $\alpha=135^{\mathrm{h}} 4^{\mathrm{m}} 57.25^{s}$, $\delta=+23^{\circ} 30^{\prime} 11.33^{\prime \prime}(\mathrm{J} 2000.0)$.

baseline snapshot imaging") technique is a useful method for detecting and imaging at moderate resolution compact emission in starbursts. ${ }^{2}$

\subsection{VLBA 13, 6, and $3.6 \mathrm{~cm}$ Follow-up}

Initially believing that our global $6 \mathrm{~cm}$ observations (see $\S 2.1$ ) had detected two new bright $\mathrm{SNe}$, we applied for and obtained

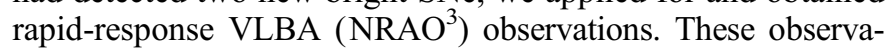
tions used dual polarization at wavelengths of 13,6 , and $3.6 \mathrm{~cm}$ at a total data rate of $256 \mathrm{Mbit} \mathrm{s}^{-1}$ and were made on 2006 January 9 (experiment BP129). To optimize $u v$ coverage, the allocated $10 \mathrm{hr}$ were split into 8 blocks of $\sim 70$ minutes. Each block was equally divided into 23 minutes at each wavelength. The data were reduced using classic AIPS. After manual flagging using SPFLG, instrumental delay and rates were removed by fringe-fitting the relatively strong (4 Jy) calibrator $\mathrm{J} 1613+3412$ and the solutions applied to the whole experiment. Atmospheric effects were removed by using phase referencing with 2 minute scans on the nearby calibrator $\mathrm{J} 1532+2344\left(0.55^{\circ}\right.$ away from Arp 220) alternating with 3.5 minute scans on the target at each wavelength (Wrobel et al. 2000; Beasley \& Conway 1995). The observed phases were very stable at all wavelengths. At $3.6 \mathrm{~cm}$ typical differences in phase between adjacent calibrator scans had rms values of $\sim 4^{\circ}$, and visual estimation of the uncertainties in interpolated phase were at a comparable level. Given the small separation between the target and the calibrator, the effect of angular variations on phase errors should be negligible, hence we do not expect any appreciable effect on source amplitudes due to phase incoherence. Amplitude calibration was based on system temperature measurements for each antenna provided as TY tables; based on previous experience with the VLBA we estimate these should be accurate to $\sim 5 \%$.

At each of the three wavelengths the task IMAGR was used to produce $2048 \times 2048$ pixel images of both the western and eastern nuclei regions with pixel spacing of 0.25 mas pixel $^{-1}$. In order to reduce imaging artifacts from poorly sampled extended emission, a minimum $u v$ distance of $10 \mathrm{M} \lambda$ was used at 6 and

\footnotetext{
2 The technique has been subsequently applied to several other sources within our COLA sample observations (see R. Parra et al. 2007, in preparation).

3 The National Radio Astronomy Observatory is a facility of the National Science Foundation operated under cooperative agreement by Associated Universities, Inc.
} 

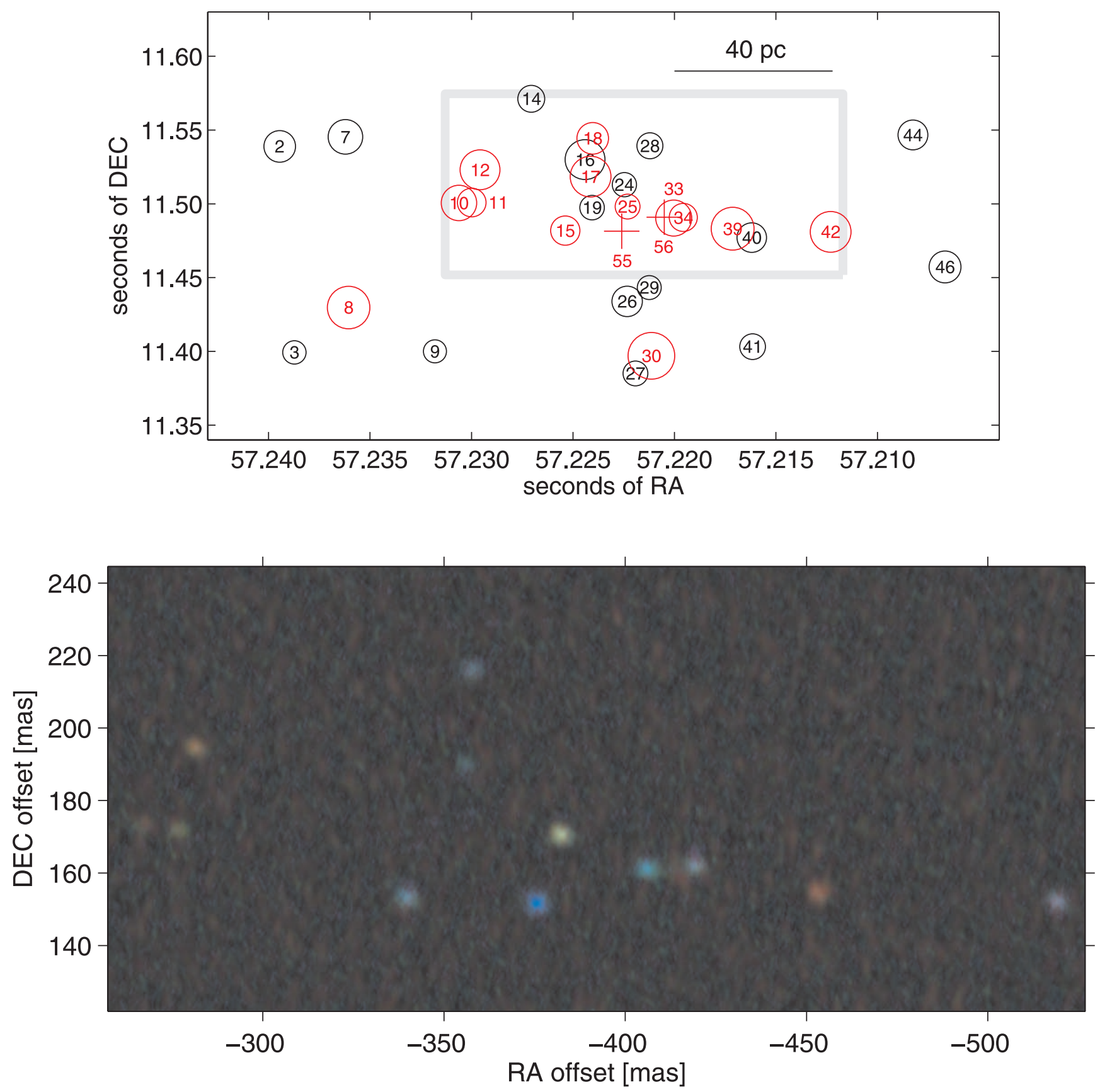

FIG. 2.-Distribution of compact radio sources in the western nucleus. Top: Detected sources at $18 \mathrm{~cm}$ wavelength shown as circles with area proportional to flux density (as reference, source number 30 is $1.23 \mathrm{mJy}$ ). The plotted numbers, prefixed by "W" for "west," are the source names as defined by Lonsdale et al. (2006), plus two new sources (W55 and W56). Sources detected at any wavelength shortward of $18 \mathrm{~cm}$ are shown in red. Red crosses indicate the positions of two previously uncataloged sources. The gray rectangle indicates the relative position of the composite image shown in the bottom panel. Bottom: Red, green, and blue composite image displaying, respectively, 13, 6, and $3.6 \mathrm{~cm}$ images from experiment BP129. The axes of this image are in mas from the reference position indicated by the cross in Fig. 1. An expanded contour image of the region around W33 is shown in Fig. 4.

$3.6 \mathrm{~cm}$. After experimentation the most sensitive image at $13 \mathrm{~cm}$ was obtained using a minimum baseline cutoff of $5 \mathrm{M} \lambda$. To maximize image sensitivity, pure natural weighting was used at each wavelength. Only a couple of hundred CLEAN components were needed before the residual image became noise-dominated. Despite the use of $u v$ limits, the final CLEAN images contained weak ripples of $\sim 20$ mas of wavelength possibly introduced by the gridding algorithm of IMAGR. In a final step these ripples were removed by taking the Fourier transform of the images, eliminating the associated spikes and inverse-Fourier-transforming back to obtain almost ripple free images. The final images at 6 and $3.6 \mathrm{~cm}$ achieved the theoretical noise, but the noise at $13 \mathrm{~cm}$ was somewhat larger (see Table 1). This may be due to the remaining effects of extended structure, or possibly the weakness of the calibrator at this wavelength.

Images of the western and eastern nuclei are presented as two RGB composite images (Figs. 2 and 3). These images were constructed by assigning to the $R, G$, and $B$ channels versions of the 13,6 , and $3.6 \mathrm{~cm}$ images produced by restoring the CLEAN components using a circular beam of 5 mas at all three wavelengths. These images clearly show that the detected sources have very different colors demonstrating their wide variety of spectral properties. 

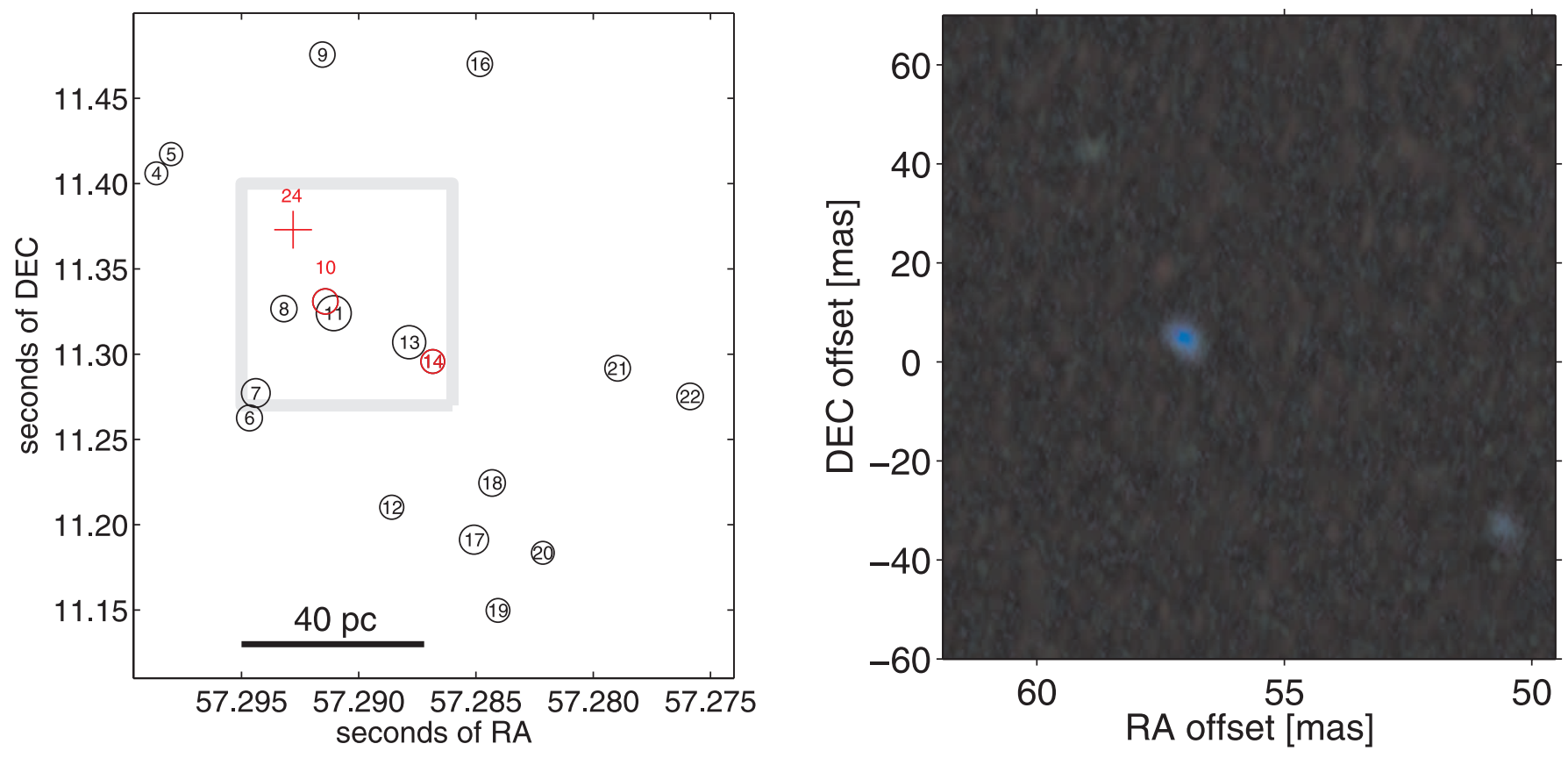

FIG. 3.-Distribution of compact radio sources in the eastern nucleus. Left: Detected sources at 18 cm wavelength shown as circles with area proportional to flux

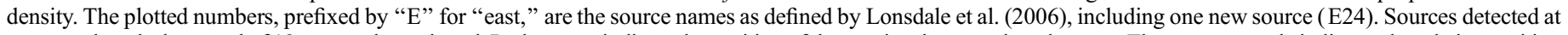

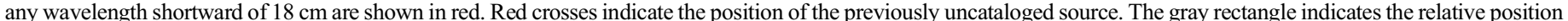

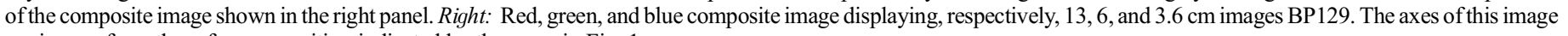
are in mas from the reference position indicated by the cross in Fig. 1.

In addition to these images, an image was made at $3.6 \mathrm{~cm}$ wavelength using uniform weighting to check for source resolution. All except one of the sources (see $\S \S 3.2$ and 5.2.3) were unresolved.

\subsection{Additional Data}

In addition to our own BP129 observations we also reduced archival $6 \mathrm{~cm}$ VLBA data from 2003 January 2 (BN022) consisting of $2 \mathrm{hr}$ at a data rate $128 \mathrm{Mbit} \mathrm{s}^{-1}$. These observations used a phase referencing cycle of 3 minutes on Arp 220 and 1 minute on the calibrator J1516+1932. These data were reduced following the procedures described in the previous section. Finally, to aid in the search for sources at high frequency and for constraining the long-wavelength part of the source spectra, we have used data from global $18 \mathrm{~cm}$ experiments GD17A (Lonsdale et al. 2006) and GD17B (H. Thrall et al. 2007, in preparation). The latter data were observed approximately 1 yr before BP129; however, most $18 \mathrm{~cm}$ light curves are known to vary slowly (Rovilos et al. 2005), so these data can be used to extend the spectra obtained from BP129. A summary of all the available data indicating the achieved sensitivities and resolutions is given in Table 1.

\subsection{Source Astrometry and Previous $6 \mathrm{~cm}$ Observations}

Comparing our $13 \mathrm{~cm}$ image from experiment BP129 with published $18 \mathrm{~cm}$ images (Smith et al. 1998), we found four bright sources with the same relative separations; however, the absolute positions of the two images differed by $\sim 100$ mas in declination. Careful checking convinced us that the positions from BP129 are correct and that it is the published positions that are wrong. In particular, $6 \mathrm{~cm}$ source positions from EP049, BP129, and BN022 all agree.

Previously published $18 \mathrm{~cm}$ VLBI continuum and spectral line images have all been made by phase-referencing to the brightest $1667 \mathrm{MHz}$ OH maser in the western nucleus (Smith et al. 1998; Rovilos et al. 2003). Absolute positions were then assigned by adding the absolute position of this maser as determined from an old MERLIN astrometric observation. The fact that we find an error in absolute position that is entirely in one coordinate and is very close to 100 mas suggests that a typographical error was made either when originally recording or later when manually copying the position of this brightest maser. Note that this astrometric error affects both the published absolute positions of $18 \mathrm{~cm}$ continuum sources and $\mathrm{OH}$ masers. It will not, of course, affect the relative positions of masers and compact $18 \mathrm{~cm}$ continuum.

A consequence of the above astrometric error is that previous searches for sources at $6 \mathrm{~cm}$ were made centered on the wrong field. However, this does not appear to be the primary reason why previous observations at $6 \mathrm{~cm}$ failed to detect anything. The $6 \mathrm{~cm}$ data from Rovilos et al. (2005) has been rereduced and the correct positions searched, but only hints of detections have been found. The reason is most likely the large angular distance $\left(5.76^{\circ}\right)$ to the calibrator combined with the fact that these observations were made near solar maximum when ionospheric effects could have been significant even at $6 \mathrm{~cm}$. In contrast, the new absolute positions derived from our BP129 VLBA observations are accurate to within a few mas (Pradel et al. 2005). It should be noted that the nearby calibrator used for these observations was not cataloged at the time of the earlier imaging attempts at $6 \mathrm{~cm}$ (Petrov et al. 2005).

\section{RESULTS AND ANALYSIS}

\subsection{Source Detection Criteria}

Visual inspection of Figures 2 and 3 shows that many sources are clearly detected at short wavelengths. However, to more rigorously define a complete list of detections, we defined quantitative detection criteria. We made two passes through the BP129 images, first searching around the positions of cataloged $18 \mathrm{~cm}$ sources and then searching the whole area of the eastern and western nuclei. In the first pass sources could be accepted as real at a lower signal-to-noise ratio than in the second pass because of 
TABLE 2

Properties of Detected Radio Sources and Measured Fluxes in $\mu$ Jy

\begin{tabular}{|c|c|c|c|c|c|c|c|c|c|c|c|c|}
\hline $\begin{array}{c}\text { NAME } \\
\text { (1) }\end{array}$ & $\begin{array}{l}\mathrm{SN} \\
\text { (2) }\end{array}$ & $\begin{array}{c}\alpha\left(15^{\mathrm{h}} 34^{\mathrm{m}}\right) \\
(\mathrm{s}) \\
(3)\end{array}$ & $\begin{array}{c}\delta\left(23^{\circ} 30^{\prime}\right) \\
(\operatorname{arcsec}) \\
(4)\end{array}$ & $\begin{array}{c}\text { GD17A } \\
(18 \mathrm{~cm}) \\
(5)\end{array}$ & $\begin{array}{c}\text { GD17B } \\
(18 \mathrm{~cm}) \\
(6)\end{array}$ & \multicolumn{3}{|c|}{ BP129 } & $\begin{array}{c}\alpha \\
(10)\end{array}$ & $\begin{array}{c}\tau_{18} \\
(11)\end{array}$ & $\begin{array}{l}S_{\mathrm{sy}} \\
(12)\end{array}$ & $\begin{array}{c}\text { Class } \\
\text { (13) }\end{array}$ \\
\hline W10 & 4 & 57.2307 & 11".5022 & 408.8 & 463.1 & 417.3 & 311.9 & 274.4 & -0.29 & 0.00 & 440.9 & $\mathrm{~L}$ \\
\hline W11 & & 57.2230 & 11."5015 & 175.4 & 379.3 & 572.6 & 382.4 & 357.5 & -0.72 & 0.68 & 989.7 & $\mathrm{R}$ \\
\hline W12 & & 57.2295 & $11 " .5244$ & 645.6 & 940.9 & 1011.4 & 458.1 & 341.0 & -0.72 & 0.00 & 989.8 & $\mathrm{R}$ \\
\hline . & 7 & 57.2240 & $11 . " 5458$ & 255.0 & 261.9 & 558.8 & 489.5 & 450.6 & -0.62 & 1.55 & 1236.2 & $\mathrm{~L}$ \\
\hline $\mathrm{W} 25 \ldots$. & & 57.2222 & $11 . " 5005$ & 98.0 & 499.9 & 1069.1 & 944.0 & 648.4 & -0.72 & 1.03 & 2249.3 & $\mathrm{R}$ \\
\hline W30.................. & 10 & 57.2214 & 11.4025 & 1228.0 & 1172.3 & 127.9 & 305.4 & -1.9 & & & & $\mathrm{~L}$ \\
\hline . & 11 & 57.2200 & 11.4910 & 428.0 & 338.3 & 582.2 & 259.3 & 396.9 & & & & $\mathrm{~L}$ \\
\hline W34 .................. & & 57.2195 & 11"4919 & 160.8 & 341.6 & 698.8 & 813.2 & 742.8 & -0.72 & 2.08 & 2339.2 & $\mathrm{R}$ \\
\hline 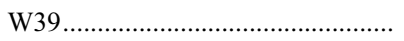 & 12 & 57.2171 & 11.4845 & 870.2 & 762.2 & 748.9 & 279.3 & 119.5 & -1.87 & 1.20 & 2475.1 & $\mathrm{~L}$ \\
\hline 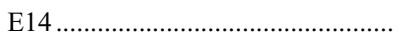 & & 57.2868 & $11^{\prime \prime} 2970$ & 62.1 & 62.0 & -100.4 & 498.1 & 549.2 & -0.72 & 8.60 & 2428.7 & A \\
\hline E24 & & 57.2928 & 11.3728 & $<27.0$ & $<27.0$ & 114.2 & 477.1 & 374.4 & -0.72 & 4.46 & 1529.6 & $\mathrm{~S}$ \\
\hline
\end{tabular}

Notes.-Col. (1): Source names from Lonsdale et al. (2006) for all sources except W55, W56, and E24, which are newly detected sources and have been named using the next available "W" and "E" numbers. Col. (2): Names used in Smith et al. (1998) and Rovilos et al. (2005). Cols. (3) and (4): J2000.0 right ascencion and declination obtained by fitting a Gaussian to the highest frequency detection in the BP129 observations. Cols. (5) and (6): $18 \mathrm{~cm}$ fluxes from Lonsdale et al. (2006) and H. Thrall et al. (2007, in preparation), respectively. Upper limits for newly detected sources are indicated at $3 \sigma$. Cols. (7), (8), and (9): 13, 6, and $3.6 \mathrm{~cm}$ fluxes from the observations of this work. The $3.6 \mathrm{~cm}$ flux for source W42 (marked by an asterisk) is given in $\mu \mathrm{Jy}$ beam ${ }^{-1}$ because this source is resolved. Col. (10): Fitted optically thin synchrotron spectral index for sources of class L (col. 13) and -0.72 for sources in all other classes (see $\S 4.2$ for details). Sources W30, W33, and E10 resulted in poorly constrained fits so no results are given. Col. (11): Fitted optical depth at $18 \mathrm{~cm}$. Col. (12): Fitted optically thin synchrotron flux at $18 \mathrm{~cm}$. Col. (13) Source variability class defined in $\S 3.3$.

the much smaller number of beam areas searched. In the first pass we searched separately at each of our three wavelengths; a source was considered a detection if it was detected at one or more wavelengths. Since sources missed in the first pass would be uncataloged at $18 \mathrm{~cm}$ (and hence very weak at both 18 and $13 \mathrm{~cm}$ ), these sources would have spectra that peak at high frequency. Therefore, in the second pass we only searched a single composite image formed by averaging our 6 and $3.6 \mathrm{~cm}$ images.

In the first pass we conservatively took the $18 \mathrm{~cm}$ beam size as our search area around each of the 49 known positions. The number of independent beam areas searched at each wavelength $(\lambda)$ was then $N_{s}=49(18 / \lambda)^{2}$. Analysis of the histograms of the images in regions away from detected sources showed distributions that were very close to Gaussian allowing the use of Gaussian statistics. Setting the detection criterion as any spike within a given search area above $\eta \sigma$ the probability that one or more entries in the list of detected sources is a noise spike is $F=1-P(I<\eta \sigma)^{N_{s}}$, where $P(I<\eta \sigma)$ is the cumulative probability of a noise spike at a given beam area being less than $\eta \sigma$. At each wavelength we chose $\eta$ such that $F$ was $0.5 \%$. The resulting detection limits at 13,6 , and $3.6 \mathrm{~cm}$ are $3.8,4.1$, and $4.4 \sigma$, respectively. Using these criteria, a total of 15 sources were detected in the first pass.

In our second pass we then looked for new sources at any other position contained within two square fields of $512 \times 512$ mas enclosing the eastern and western nuclei (see $\S 2.2$ ). As explained above we searched a composite image made by averaging the 6 and $3.6 \mathrm{~cm}$ images. This time the area searched was much larger than in the first pass, therefore for a $0.5 \%$ confidence of false detection the required detection limit was $5.1 \sigma$. In this second pass we detected three new sources (two in the west). In total, despite our rather modest sensitivity, we detected a total of 18 sources (see Table 2). Given that in the first pass three separate searches were made (one at each wavelength), and one search done in the second pass, all with $0.5 \%$ confidence of false detection, we estimate an overall $2 \%$ probability that one or more of the sources in Table 2 is a false detection.

It should be mentioned that the BP129 image at $3.6 \mathrm{~cm}$ shows several pointlike features that lie just below our detection threshold and are mostly located in the region with the highest density of sources (within 20 mas of position $(-380,160)$ mas in the bottom panel of Fig. 2). Almost certainly some of these features will be confirmed as detections in higher sensitivity observations.

\subsection{Source Structure}

The only resolved source at $3.6 \mathrm{~cm}$ is W42. This source is located $\sim 100$ mas to the west of the region with the highest surface density of sources (see Fig. 2). Along the position angle corresponding to the beam minor axis the FWHM of W42 is about twice as big as the beam FWHM (see Fig. 4, left). The fact that other sources were unresolved shows that the resolution of W42 is not an artifact of residual phase errors in the data. Another source deserving special attention is W33, which, in addition to its peculiar spectrum (see Fig. 6), shows possible signs of an elongated structure in the northsouth direction at $13 \mathrm{~cm}$ (see Fig. 4, right). This source is tightly located between two bright sources (W56 and W34) and lies near the edge of a patch of diffuse $18 \mathrm{~cm}$ emission covering the central region of the western nucleus ( $\mathrm{H}$. Thrall et al. 2007, in preparation). We speculate about the nature of this source in $\S$ 5.2.4

\subsection{Source Variability}

In order to search for correlations between source spectral properties and source age, we classify our detected sources in terms of their broad $18 \mathrm{~cm}$ variability properties. A more detailed variability study including detailed light-curve modeling will be 

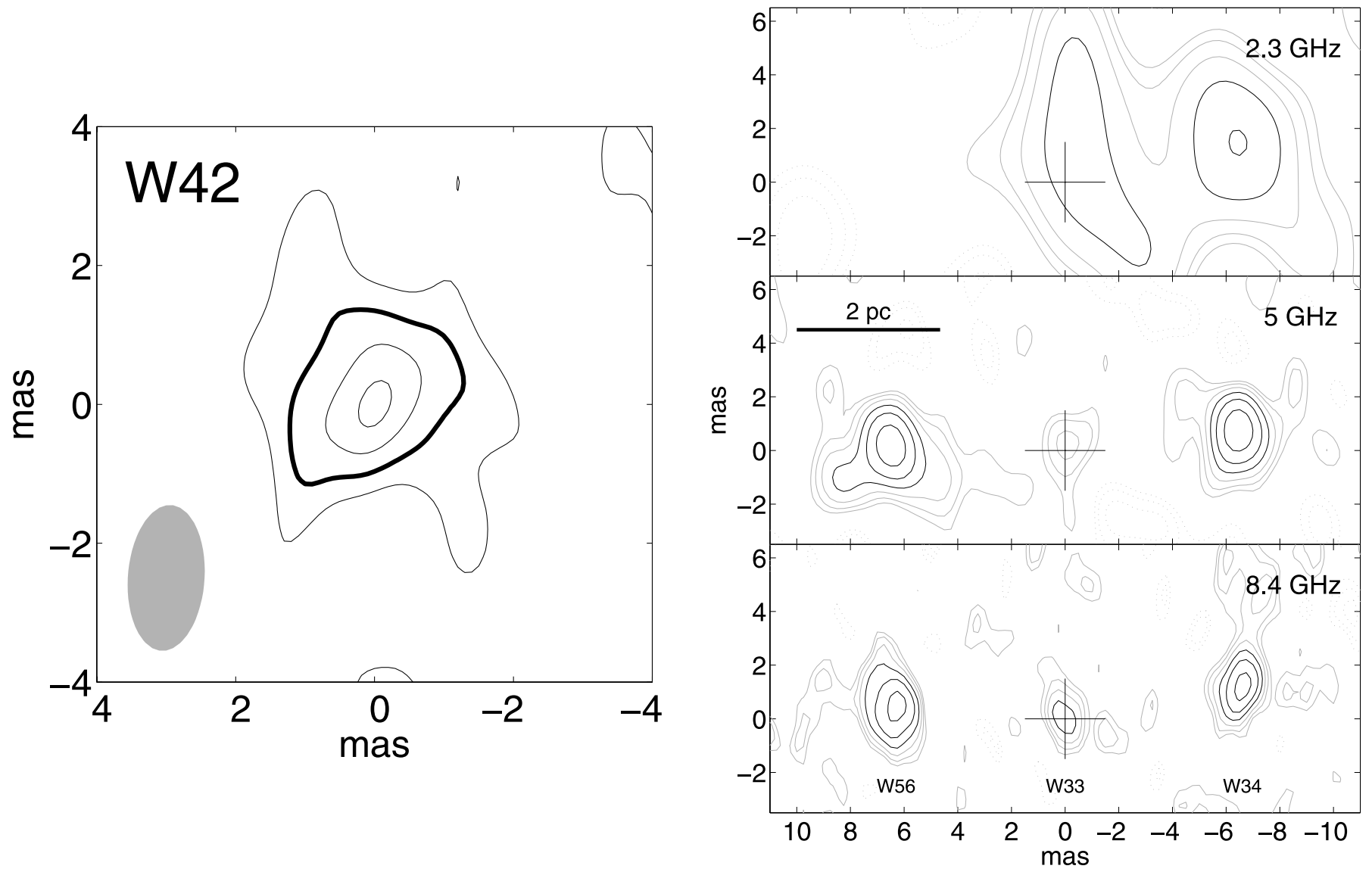

FIG. 4.-Detailed structure of source W42 and of the W33 region. Left: Contour map of W42 produced from the $3.6 \mathrm{~cm}$ wavelength uniformly weighted image. Contour levels are $-25,25,50,75$, and 95 percent of the peak (see Table 2). The $50 \%$ contour is drawn with a thick line, and the $-25 \%$ ones with dashed lines (none visible). The $50 \%$ contour appears elongated in the northeast-southwest direction with respect to the beam (bottom left corner) indicating source resolution. Right: Contour maps of the W33 region at 13,6 , and $3.6 \mathrm{~cm}$. Contour levels are ...-2, $-\sqrt{2}, \sqrt{2}, 2 \ldots$, multiplied by the corresponding map noise (see Table 1). Contour levels below the detection threshold at every frequency are drawn in gray. Negative contours are drawn with dotted lines. The axes are in mas relative to the peak in the $3.6 \mathrm{~cm}$ map, whose position is indicated by a cross in all three panels (see Table 2).

presented in a subsequent paper (Thrall et al. in preparation). We define four classes of sources:

Class L (long-lived). These are eight sources already detected by Smith et al. (1998) based on observations in 1994; hence these sources are at least $11 \mathrm{yr}$ old. In addition, analysis of the first $6 \mathrm{yr}$ of monitoring data by Rovilos et al. (2005) showed that all these sources had slow or undetectable decays of their $18 \mathrm{~cm}$ flux densities and are therefore likely to be significantly older.

Class $R$ (rising).-These are four sources not detected by Rovilos et al. (2005) but which are detected in the latest $18 \mathrm{~cm}$ epochs at flux levels significantly above the Rovilos et al. (2005) detection limits. W11, W25, and W34 were present in GD17A but not in the $18 \mathrm{~cm}$ experiment (GL26B) made 12 months earlier (Lonsdale et al. 2006). Source W12 was present in both GD17A and GL26B but not at earlier epochs (Lonsdale et al. 2006).

Class $S$ (single-epoch).-These are three sources only detected in our new high-frequency data and not in any $18 \mathrm{~cm}$ epoch.

Class A (ambiguous).- These are sources with ambiguous variability classification. For instance one of the three sources in this class (W15) is detected in both GD17A and GD17B at similar flux densities, but at levels below the Rovilos et al. (2005) detection limits; therefore, it could be either a new source or a long-lived stable source not detected earlier because of sensitivity limitations. Similar considerations apply to source E14. Source E10 was not detected by Rovilos et al. (2005); it was detected in GD17A, but not in GD17B.
In addition to the above classification scheme, it is also interesting to consider possible variations in $6 \mathrm{~cm}$ flux densities by comparing our data with the BN022 data taken just over $2 \mathrm{yr}$ earlier. Considering the thermal noise on both epochs only two sources show $>2 \sigma$ variations, namely, W18 $(-625 \mu \mathrm{Jy})$ and W33 ( $-505 \mu \mathrm{Jy})$. Surprisingly, both are class L sources. W33 has other unusual properties in addition to its variability and is discussed further in $\S 5.2 .4$.

\subsection{Source Spectra}

To determine the spectra of our detected sources, we first defined a source position based on the highest frequency detection; then, at this pixel we determined the brightness at each of the three simultaneously observed wavelengths $(13,6$, and $3.6 \mathrm{~cm})$. Tests fitting Gaussians to the four brightest sources at 3.6, 6, and $13 \mathrm{~cm}$ showed consistent positions at the three wavelengths to within $\sim 0.5$ pixels in each coordinate ( 1 pixel being 0.25 mas). Since the source position is defined at $3.6 \mathrm{~cm}$, the maximum impact of any registration offset on the derived spectra is set by its effect at $6 \mathrm{~cm}$. At this wavelength the map registration uncertainty amounts to less than $10 \%$ of the beam minor-axis FWHM and will contribute only a $2 \%$ error on the amplitude, which is negligible relative to the map noise. The resulting source flux densities using the above procedure are given in Table 2. In some cases the reported numbers are less than the noise or are negative, but these measurements are still useful for constraining spectral 


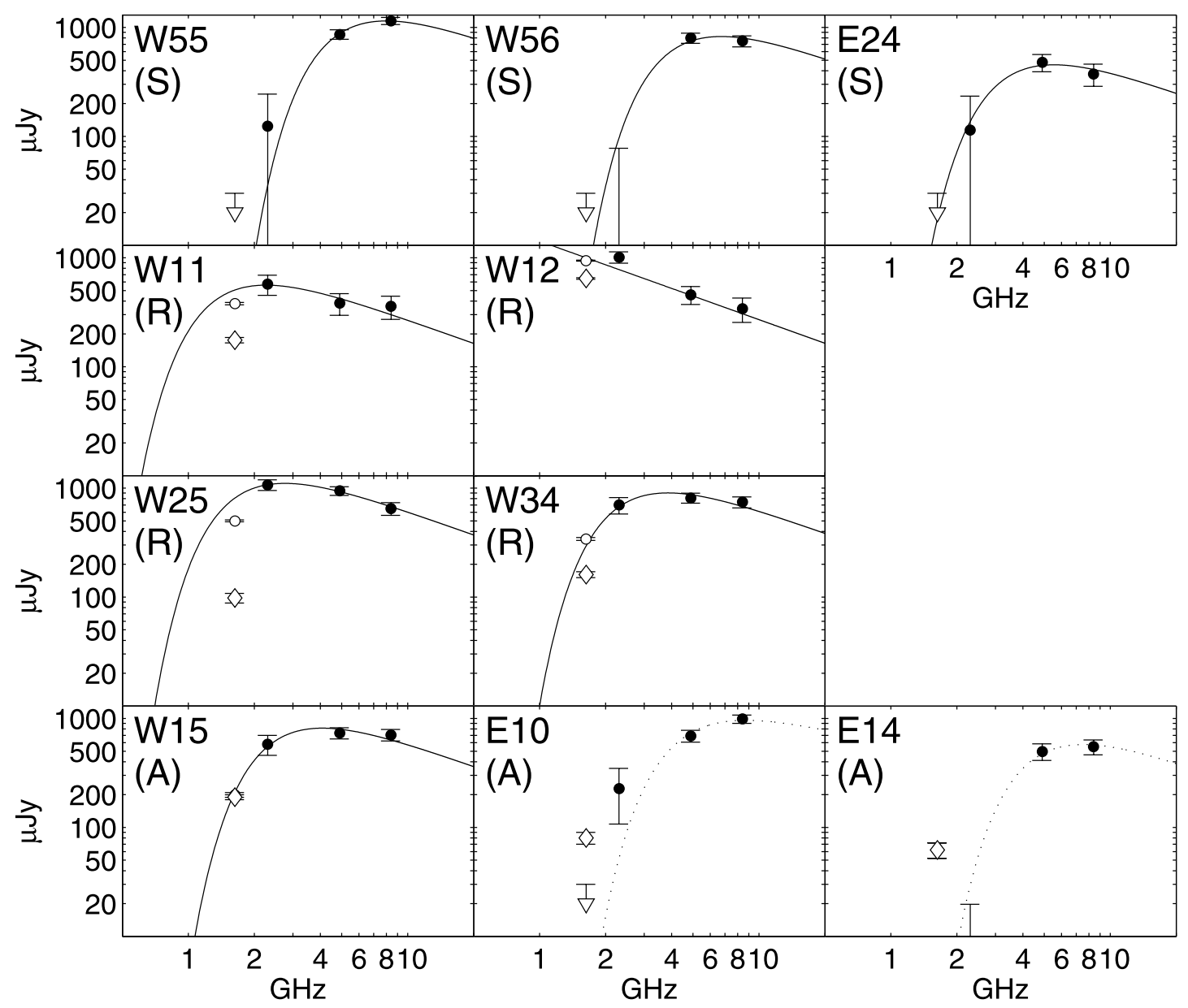

Fig. 5.-Spectra and models of short-wavelength detected sources not in the original $18 \mathrm{~cm}$ sample of Smith et al. (1998); these sources are either new since 1995, weak at long wavelengths, or both. The variability subclass to which each source belongs ( R, S, and A; see $\S 3.3$ for definition) is indicated in the top left corner of each spectrum. The data at $13 \mathrm{~cm}(2.3 \mathrm{GHz}), 6 \mathrm{~cm}(5 \mathrm{GHz})$, and $3.6 \mathrm{~cm}(8.4 \mathrm{GHz})$ were taken from simultaneous observations in BP129 and are shown as filled circles. For those points error bars are plotted at $\pm 1 \sigma$ about the measured value given in Table 2 . In cases where the measurement gives a negative flux density, only the upper error bar is plotted. The $18 \mathrm{~cm}$ data comes from the earlier epochs of GD17A and GD17B, shown as diamonds and open circles, respectively. In the case of detections at this wavelength error bars are plotted at $\pm \sigma$. If a source is undetected at one or more of the $18 \mathrm{~cm}$ epochs, an upper limit is plotted. The curves shown are the best fits of the model spectrum described in $\S 4.1$ obtained by fitting only to the simultaneous 13,6, and $3.6 \mathrm{~cm}$ data from the BP129 epoch. For sources E10 and E14 the best fits are shown with dotted lines, indicating that these models were unable to fit all the data within the error bars.

fits (see $\S 4.2)$. An exception to the above procedure is the resolved source W42 (see $\S 5.2 .3$ ), for which the integrated flux density at $3.6 \mathrm{~cm}$ was determined within a box containing the source.

For the $18 \mathrm{~cm}$ flux densities a slightly different procedure was followed because these data were not taken simultaneously with the rest of the wavelengths and so exact position registration at the pixel level could not be assured. GD17A fluxes are taken from Lonsdale et al. (2006) in the case of tabulated detections; otherwise the map was searched at the detected high-frequency position within the $18 \mathrm{~cm}$ beam area for anything above $3 \sigma$. In no cases was any new detection found; these sources are reported in Table 2 as $3 \sigma$ upper limits. For GD17B the largest peak within an $18 \mathrm{~cm}$ beam area of the high-frequency position was located, and in most cases large SNR detections were found. In cases with no peak above $3 \sigma$ these upper limits are reported in Table 2 .

All the measured spectra for sources in classes R, S and A are displayed graphically in Figure 5. Sources in class L are displayed in Figure 6. Taken together, a wide variety of spectral shapes are seen from inverted to flat to steep. Overall the distribution of spectra are very similar to those of the compact sources detected in knot A of the starburst Arp 299 (see Neff et al. 2004). Further discussion and modeling of our observed Arp 220 spectra is presented in $\S 4$.

\subsection{Source Spatial Distribution}

An initial impression from Figure 2 is that the 15 shortwavelength detections in the western nucleus (red circles in the top panel) have approximately the same spatial distribution as the $18 \mathrm{~cm}$ sources from Lonsdale et al. (2006). However, if we only consider sources not seen in Smith et al. (1998) (i.e., classes $\mathrm{R}, \mathrm{S}$, and A) which have rising or peaked spectra (see Fig. 5), these appear to be more centrally concentrated. All seven such sources are found within the gray rectangle in Figure 2. In contrast, among the $18 \mathrm{~cm}$ sources of Lonsdale et al. (2006) the fraction inside the rectangle is only $17 / 29=0.58$. If the class $\mathrm{R}, \mathrm{S}$, and A sources have the same spatial distribution there is only a $2.4 \%$ probability of all being within the rectangle. However, this is only an a posteriori statistic and so must be treated with caution. Future observations plus robust statistical tests are needed to check it. Note that even if true, it does not necessarily imply a difference in spectral properties with position. These results might be explained by differences in overall luminosity combined with the present high flux detection threshold at short wavelength. Supporting this explanation is the fact that the median $18 \mathrm{~cm}$ luminosity is a factor of 2 larger inside the rectangle than outside. Furthermore, a Kolmogorov-Smirnov test finds only a $6 \%$ probability that the 


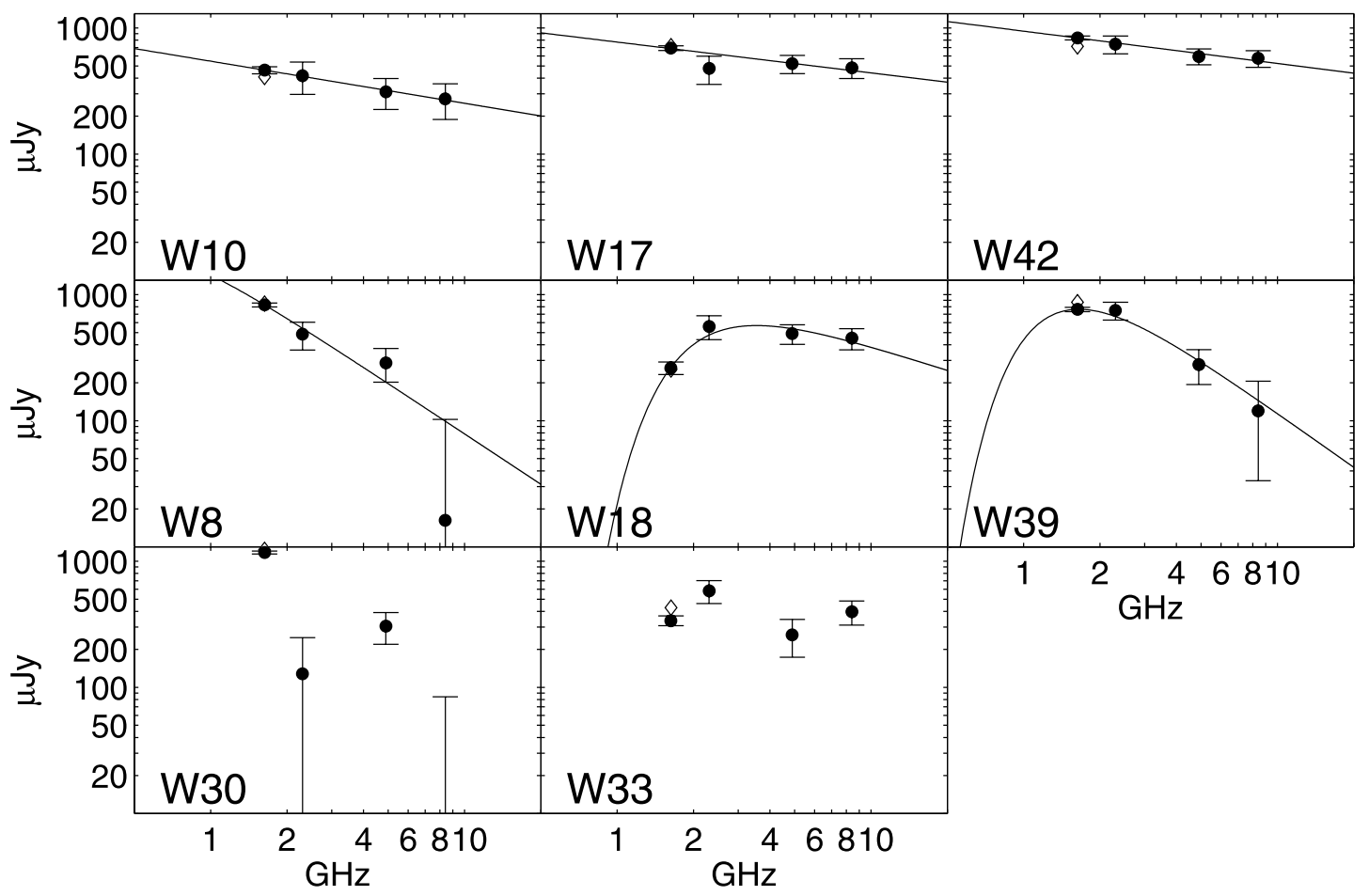

FIG. 6.- Spectra and models for the short-wavelength sources detected in the original $18 \mathrm{~cm}$ sample of Smith et al. (1998) (class L sources; see $\S 3.3$ ). In all cases error bars are plotted at $\pm 1 \sigma$ about the measured value given in Table 2 . When the measured flux density is negative, only the upper error bar is plotted. The first row shows three sources with relatively flat spectra. The middle row shows the remaining sources that are well fitted by a power-law synchrotron plus FFA model (see $\S 4.1$ ). Finally, the last row shows two sources for which good fits could not be obtained. The data at $13 \mathrm{~cm}(2.3 \mathrm{GHz}), 6 \mathrm{~cm}(5 \mathrm{GHz})$, and $3.6 \mathrm{~cm}(8.4 \mathrm{GHz})$ were taken from simultaneous observations in BP129. The $18 \mathrm{~cm}$ data from earlier epochs GD17A and GD17B are shown as filled circles and open diamonds, respectively (in some cases these data points overlie each other). Error bars are shown at $\pm \sigma$ (see Table 1) The curves shown are the best fits of the synchrotron plus FFA model spectrum described in $\S 4.1$ as fitted to the data at all four wavelengths. For sources W30 and W33 no reasonable fits could be obtained and therefore no model is plotted.

$18 \mathrm{~cm}$ sources inside and outside are drawn from the same flux density distribution.

\section{ANALYSIS OF SOURCE SPECTRA}

\subsection{Cause of Low-Frequency Spectral Turnovers}

The majority of the spectra shown in Figure 5 are consistent with power-law synchrotron spectra with turnovers below some critical frequency. Such spectra are typically observed in both SNe and SNRs (Weiler et al. 2002; Allen \& Kronberg 1998). In principle, the low-frequency turnovers could be due to either synchrotron self-absorption (SSA) or free-free absorption (FFA). Although the former has been successfully invoked for weak Type II and Type Ib/c SNe (Soderberg et al. 2005), in all successfully modeled powerful Type II SNe and SNRs FFA is the dominant process (Weiler et al. 2002). This is confirmed by the fact that for those powerful SN and SNR sources that are resolved, the brightness temperatures are too small for SSA to operate.

For SSA to occur at a given frequency, a characteristic relationship between source luminosity and diameter is required (Chevalier 1998). For a given shell expansion speed this implies a relationship between peak luminosity and rise time. Inversely, for an observed combination of these parameters there is an associated expansion speed required for SSA to dominate (see Fig. 4 in Chevalier 1998).

Sources of class $\mathrm{R}$ have spectra peaking around $4 \mathrm{GHz}$ and peak luminosities of $\sim 10^{28} \mathrm{ergs} \mathrm{s}^{-1} \mathrm{~Hz}^{-1}$. If a rise time at $4 \mathrm{GHz}$ of over $2 \mathrm{yr}$ is assumed (the time between GD17A detections and BP129), then these sources lie in the region of the luminosityrise time diagram occupied by Type IIn SNe. In turn, for SSA to apply in these sources, the required expansion velocities are
$<3000 \mathrm{~km} \mathrm{~s}^{-1}$ which is considered too slow for this kind of SN (Chevalier 1998). The longer lived class L sources with spectral turnovers would require even slower expansion velocities and so are even more unlikely to be affected by SSA. We have no idea of the age or rise times of class $\mathrm{S}$ sources. In principle these could be rare luminous Type $\mathrm{Ib} / \mathrm{c}$ sources with fast rise times and hence could be affected by SSA. However, it seems improbable that we detect four such sources given the short time their luminosities peak. More likely these are class R sources observed at an earlier evolutionary stage. We conclude that SSA is unlikely to be a significant cause of the low-frequency cutoff in any of our sources. We will be able to test this assumption using future observations over a wide frequency range by examining the shape of the lowfrequency cutoff; unfortunately the present sparse nonsimultaneous spectral data does not allow such a test.

\subsection{Free-free Absorption Model}

In this section we fit the observed spectra as a generic power law plus FFA. We leave to $\S 5.1$ the question of whether the data are more consistent with SNe or SNRs. In SNe the FFA occurs mostly in the wind-blown bubble from the progenitor star. In contrast, in SNRs the FFA is due entirely to the foreground ionized ISM. The model spectra we fitted are described by

$$
S_{\nu}=S_{\mathrm{sy}}(\nu / 1.63)^{\alpha} \exp \left[-\tau_{18}(\nu / 1.63)^{-2.1}\right],
$$

where $\nu$ is the frequency in $\mathrm{GHz}, S_{\mathrm{sy}}$ is the $18 \mathrm{~cm}$ unobscured synchrotron flux in $\mu \mathrm{Jy}, \alpha$ is the optically thin nonthermal spectral index, and $\tau_{18}$ is the optical depth at $18 \mathrm{~cm}$. This model corresponds to all the FFA being foreground to the synchrotron emission and all parts of the source being subject to the same foreground opacity. 
Note the latter is not true if the FFA absorbing medium is clumpy with clump scale sizes smaller than the emitting source, in which case more complex models will apply (Natta \& Panagia 1984; J. E. Conway et al. 2007, in preparation).

The fitting proceeded by finding for each source the global minimum of the $\chi^{2}$ metric in a gridded three-dimensional volume. To improve accuracy after an initial coarse search, a second search was done using a denser grid around the coarse solution. We note that for some sources at some wavelengths the measured flux densities were negative. However, these values are formally our best estimates and therefore were considered in the fitting procedure. This is a simpler and less potentially biased procedure than fitting a mixture of measurements and upper limits.

For the class L sources we fitted all three free parameters of the model to the four spectral data points provided by the three wavelengths in BP129 plus $18 \mathrm{~cm}$ data from the nonsimultaneous GD17B observations (taken $0.84 \mathrm{yr}$ before). The use of the nonsimultaneous $18 \mathrm{~cm}$ data in the spectral fitting is justified for the class L sources because they are known to have low variability at this wavelength (Rovilos et al. 2005). Furthermore, in all cases the GD17A and GD17B measurements are consistent within the error bars, confirming low recent variability (compare diamonds and circles at $18 \mathrm{~cm}$ in Fig. 6).

Solutions fitting the data within the error bars (reduced $\chi^{2}<$ 1.5) were obtained for six of the eight class L sources, the exceptions being W30 and W33. The optimum values for $S_{\mathrm{sy}}, \alpha$, and $\tau_{18}$ are summarized in Table 2 , and the corresponding model spectra are overlayed on the data in Figure 6. Note that in some cases the best solution had a slightly negative opacity $\tau_{18} \approx-0.1$, which is clearly unphysical. In those cases we set $\tau_{18}=0$ and found the values for the other parameters that minimized $\chi^{2}$; in all such cases the final $\chi^{2}$ was only slightly increased (by $\lesssim 0.5$ ). In addition to finding the optimum values, we searched the region of $\tau_{18}$ and $\alpha$ around the best fit, finding for each combination the optimum $S_{\text {sy }}$ and recording $\chi^{2}$. The area over which this $\chi^{2}$ increased by less than 1 compared to the global minimum was used to define a $68 \%$ confidence ellipse around the $\tau_{18}$ and $\alpha$ solution. These confidence ellipses are plotted in the bottom panel of Figure 7 and show in most cases a significant correlation between the solutions for the two parameters.

For the class S, R, and A sources long-term monitoring data at $18 \mathrm{~cm}$ is not available and we cannot assume that the GD17B data approximates the $18 \mathrm{~cm}$ flux density at the time of the BP129 observations. In fact, in many cases comparison of GD17A and GD17B data suggests significant variability. Fitting a more complex time-variable model adds more unknowns, and we defer such an attempt to a later paper when we have more data. Instead, here we choose to fix $\alpha$ at a value of -0.72 , corresponding to the median synchrotron spectral index of the 9 well-studied Type II SNe from Weiler et al. (2002) and then fit the remaining parameters $\tau_{18}$ and $S_{\text {sy }}$ to the three simultaneous frequency data points from BP129. We chose to do this because fitting the full threeparameter model to only three data points resulted in large uncertainties and in some cases unphysical optimum solutions that were clearly dominated by noise. Note that the above fitting method can only hope to show consistency with the assumed spectral in$\operatorname{dex} \alpha$ but does not prove it.

Using the above procedure, good fits passing through the three simultaneous frequencies and consistent with a reasonable evolution of the $18 \mathrm{~cm}$ flux densities are obtained for 8 of the 10 sources (see Fig. 5). The exceptions are E14 and E10. The fit for E14 passes through the fitted data points but predicts an $18 \mathrm{~cm}$ flux density very different from the consistent values seen in GD17A and GD17B. In E10 the fit is a little too steep to fit the $13 \mathrm{~cm}$ data point; on the other hand, such a fit is consistent with the apparently rapidly falling $18 \mathrm{~cm}$ flux density, which may point to a problem with the $13 \mathrm{~cm}$ data point. For the eight well-fitted sources the optimum values of $\tau_{18}$ obtained are shown in the top panel of Figure 7 including $1 \sigma$ error bars.

\subsection{Fitted Spectral Indices and Opacities}

Figure 7 plots the fitted $\alpha$ versus $\tau_{18}$ using different symbols for the different variability classes defined in $\S 3.3$. This figure shows that the highest opacities $\tau_{18}>4$ all occur in class $\mathrm{S}$ sources, which are those that are newly detected. There is only one class A source giving a good fit, and this also has quite a large $\tau_{18}=2.4$. Of the class $\mathrm{R}$ or rising sources 3 out of 4 have $0.7<\tau_{18}<2.1$. Finally, among the long-lived class L sources, four out of six have spectra consistent with zero opacity, There appears therefore to be a rough correlation of decreasing opacity with source type from $\mathrm{S}$ to $\mathrm{R}$ to $\mathrm{L}$. Although the exact value of $\tau_{18}$ is model dependant and Figure 7 combines sources with 2 and 3 parameter fits, the results help quantify the visual impression given from the data in Figures 5 and 6 of decreasing turnover frequency along the above sequence. In $\S 5$ we discuss this turnover/opacity correlation in terms of decreasing opacity with source age, consistent with a source evolution from young SNe to more evolved SNRs.

For the class L sources there was enough data to allow us to fit for $\alpha$ (see $\S 4.2)$. This fitting gives three sources with flat spectra $(\alpha>-0.5)$, one with intermediate spectral index $(\alpha \approx-0.6)$ and finally two with steep spectra $(\alpha<-1)$. Another source falling in this last category is W7, which is not listed in Table 2 because it was not detected at any of the BP129 wavelengths; this nondetection, however, implies that it has a very steep spectrum $(\alpha<-1.5)$.

\section{DISCUSSION}

\subsection{Supernovae or Supernova Remnants?}

An important question is whether the compact radio sources in Arp 220 are primarily SNe (interacting with their progenitor's CSM) or SNRs (interacting with the denser ISM). Here we discuss the radio properties expected from these two cases and compare with past and present observations.

\subsubsection{Background}

Despite the extreme environment in the nuclei of Arp 220 and other ULIRGs it is expected that wind-blown bubbles with a density profile $\rho_{w}(r)=\rho_{o} r^{-2}$ will still form around progenitor stars (Chevalier \& Fransson 2001; Arthur 2006). Hence there should remain a distinction between the SN phase, when the blast wave transits this declining density wind, and the SNR phase in a constant-density ISM. Equating the ram pressure of the wind with the external ISM pressure the expected bubble radius is

$$
r_{b}=0.2 \dot{m}_{-4}^{0.5} v_{w 1}^{0.5} p_{7}^{-0.5} \mathrm{pc}
$$

where $\dot{m}_{-4}$ is the mass-loss rate in units of $10^{-4} M_{\odot} \mathrm{yr}^{-1}, v_{w 1}$ is the wind speed in units of $10 \mathrm{~km} \mathrm{~s}^{-1}$, and $p_{7}$ is the interstellar pressure in units of $10^{7} \mathrm{~K} \mathrm{~cm}^{-3}$. During the initial SN phase, the shockwave moves through the stellar wind and the optically thin radio luminosity of the synchrotron-emitting shell at a fixed radius is proportional to $\rho_{o}^{k}$ or $\left(\dot{m}_{-4} / v_{w 1}\right)^{k}$, where $k$ is in the range 1.4-2 (Chevalier et al. 2006). It follows that there is a very wide range in radio luminosity for $\mathrm{SNe}$ depending on the wind properties (see Fig. 8). The most luminous RSNe with the densest winds are optically classified as Type IIn, followed by luminous Type IIL/b classes (for a recent review of SN types and their radio emission 


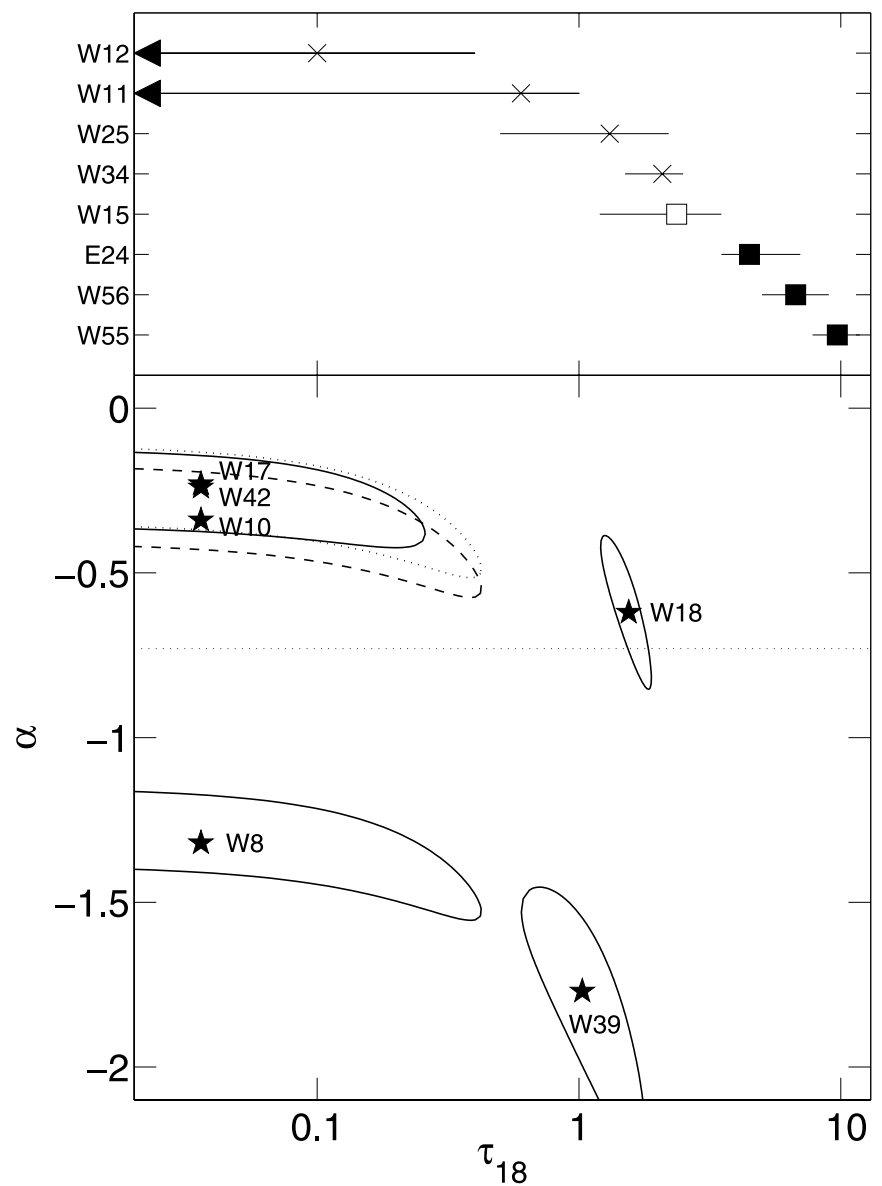

FIG. 7.- Summary of spectral model fitting results for the power law plus free-free absorption model presented in $\S 4$ 4.2. Bottom: The stars indicate the bestfit values of $\tau_{18}$ and $\alpha$ for the six class L sources with three parameters fits to four wavelength spectral data. Note that for sources W8, W10, W17, and W42 the optimum value of $\tau_{18}$ is estimated as zero, which cannot be plotted on a logarithmic scale, and therefore these points are plotted at an arbitrary small value of $\tau_{18}$. The surrounding contour around each plotted point delineates the region in which the reduced $\chi^{2}$ increases by less than 1 compared to the best fit, indicating the $68 \%$ confidence region. ( The contours for W17 and W10 are drawn in dotted and dashed lines, respectively.) The dotted horizontal line is plotted at the value of spectral index assumed for the two parameter fits shown in the top panel. Top: Fitted $\tau_{18}$ for those sources fitted using only the three wavelengths from BP129 and fixing synchrotron spectral index $\alpha=-0.72$ (see $\S 4.2$ for details). Lines indicate $1 \sigma$ error bars. Sources from variability classes $\mathrm{S}, \mathbf{R}$, and A are indicated as filled squares, crosses, and empty squares, respectively.

see Chevalier 2006). Such luminous objects are thought to be quite rare in galactic disks. For instance, Cappellaro et al. (1997) estimate that only $2 \%$ of core-collapse SNe are of the Type IIn subclass. However, there are significant uncertainties on the fraction of SN types (Chevalier 2005), and these fractions may be strongly dependent on the number of close binaries (Nomoto et al. 1996), which could be radically different in dense ULIRG nuclei.

During the SN phase the radio light-curves are defined by competition between decaying synchrotron emission and decreasing FFA, giving rise to light-curve peaks that occur progressively later at longer wavelengths (Weiler et al. 2002).

Sources are expected to enter the SNR phase, and in most cases start to brighten, when the blast wave reaches the edge of the bubble given by equation (2). The evolution just after this will be complicated as the blast wave transits shocked wind and possibly (partially) collapsed $\mathrm{H}$ II region gas (van Marle et al. 2004; Chevalier et al. 2004). Nevertheless, it is expected that radio emission will peak (Cowsik \& Sarkar 1984; Huang et al.
1994) at the beginning of the Sedov phase (i.e., when the sweptup mass, dominated by the dense surrounding ISM gas, equals the ejecta mass), which occurs at radius

$$
r_{s} \simeq 4.1 \times\left(M_{\mathrm{ej} 1} / n_{\mathrm{ISM}}\right)^{1 / 3} \mathrm{pc}
$$

where $M_{\mathrm{ej} 1}$ is the ejecta mass in units of $10 M_{\odot}$ and $n_{\mathrm{ISM}}$ is the ISM number density in $\mathrm{cm}^{-3}$. For $n_{\mathrm{ISM}}=10^{4}$ and $M_{\mathrm{ej} 1}=0.5$, the predicted size is $r_{s}=0.15 \mathrm{pc}$. Once the Sedov phase is reached the radio luminosity is expected to gradually decrease in a way consistent with the surface brightness-diameter $(\Sigma-D)$ relation for SNRs (Huang et al. 1994). ${ }^{4}$ Substituting $r_{s}$ into this relation, we obtain a rough estimate of the peak $3.6 \mathrm{~cm}$ SNR luminosity of a young SNR of

$$
L_{3.6} \approx 2.6 \times 10^{24}\left(M_{\mathrm{ej} 1} / n_{\mathrm{ISM}}\right)^{-0.53} \mathrm{ergs} \mathrm{s}^{-1} \mathrm{~Hz}^{-1}
$$

Eventually, when the internal SN gas cools to below $10^{6} \mathrm{~K}$, energy losses to atomic lines become significant (Draine \& Woods 1991) and the radio luminosity decays more rapidly than in the Sedov phase. If the external density is very large $\left(n_{\mathrm{ISM}}>10^{5} \mathrm{~cm}^{-3}\right)$, then a source can become radiative before it enters the Sedov phase (Wheeler et al. 1980), in which case it will never reach the $\Sigma-D$ relation.

In the model of Huang et al. (1994) the SNRs all lie close to the same $\Sigma-D$ (or luminosity- $D$ ) relation because they all release approximately the same kinetic energy $\left(10^{51} \mathrm{ergs}\right)$ and a constant fraction of this energy is injected into relativistic particles. The more detailed modeling of Berezhko \& Völk (2004) confirms that in the early Sedov phase, for a given diameter $D$, the radio luminosity depends only on the SN energy and is independent of the external density. We therefore expect that all core-collapse $\mathrm{SNe}$ would evolve eventually to join the SNR luminosity- $D$ relation at some diameter defined by equation (3). Hence for an object like SN 1980K (see Fig. 8) we can expect a dramatic brightening when the blast wave encounters the high-density ISM and it enters the SNR phase.

An interesting possibility is that the observed new $18 \mathrm{~cm}$ radio sources in Arp 220 (Rovilos et al. 2005; Lonsdale et al. 2006) could be such SN/SNR transition objects rather than SNe. Radio luminosity would start increasing when the blast wave reached the edge of the wind-blown bubble and would peak when the swept-up ISM gas mass equalled that of the ejecta, hence characteristic rise times would be of order $\left(r_{s}-r_{b}\right) / v_{\text {ejecta. }}$. For $v_{\text {ejecta }}=$ $10^{4} \mathrm{~km} \mathrm{~s}^{-1}$ a high external density of $n_{\mathrm{ISM}}=10^{5} \mathrm{~cm}^{-3}$ and a pressure of $p_{7}=1$ the rise time would take $\sim 7 \mathrm{yr}$, only somewhat longer than $\mathrm{SNe}$ rise times at $18 \mathrm{~cm}$. However, since for an emerging SNR the reason for the luminosity increase is quite different than for a SN (a general increase of high-energy particles and field rather than reduced FFA), such objects are expected to show very different multiwavelength light curves. According to the model of Berezhko \& Völk (2004) the radio spectrum is expected to keep approximately the same shape during the rise phase and hence we would expect the multiwavelength lightcurves to show similar behavior, in contrast to the characteristic delay between wavelengths observed in SNe.

At the other end of the SN luminosity range (see Fig. 8) for objects like SN 1986J and SN 1979C the observational data are consistent with a fairly smooth luminosity transition between the

\footnotetext{
4 Although recent analysis (Urošević et al. 2005) casts doubt on whether a physically significant $\sigma-D$ relation exists for most SNR in our own and nearby galaxies, such a correlation is confirmed for M82 and possibly also for SNRs in dense environments within our galaxy.
} 
SN and SNR phases. This might be expected because the progenitors of such powerful RSNe have the densest winds and therefore the smallest density contrasts between their terminating wind and the external medium.

\subsubsection{Previous Observations}

In their discovery paper, Smith et al. (1998) proposed an SN-based model for the compact sources in Arp 220 in which all were due to extremely luminous Type IIn SNe. The number of observed sources and the total far-IR (FIR) luminosity were found to be consistent if all these $\mathrm{SNe}$ were as radio luminous as the Type IIn supernova SN 1986J. Chevalier \& Fransson (2001) argued that this model was unlikely because such luminous objects comprise only a small fraction of core-collapse SNe. Observational doubts on the Smith et al. (1998) model were cast by the $18 \mathrm{~cm}$ light-curve monitoring of Rovilos et al. (2005), which did not show the decay in flux expected from SN 1986J-like sources. Consequently, both Rovilos et al. (2005) and Lonsdale et al. (2006) have suggested that most sources in Arp 220 are instead higher luminosity versions of the SNRs in M82.

The above SNR hypothesis seems plausible if we consider the expected and observed flux densities. In equation (4) we estimated the maximum luminosity of an SNR at wavelength $3.6 \mathrm{~cm}$ as a function of ISM density. Assuming a spectral index of -0.7 and scaling to the distance to Arp 220 this predicts $18 \mathrm{~cm}$ flux densities of $1.2 \times\left(M_{\mathrm{ej} 1} / n_{\mathrm{ISM}}\right)^{-0.53} \mu \mathrm{Jy}$. For the median $18 \mathrm{~cm}$ source flux density in the western nucleus of $207 \mu \mathrm{Jy}$ (Lonsdale et al. 2006) and assuming $M_{\mathrm{ej} 1}=0.5$, we obtain an estimated ISM density of $n_{\text {ISM }}=8300 \mathrm{~cm}^{-3}$, which is consistent with the mean molecular density of $1.5 \times 10^{4} \mathrm{~cm}^{-3}$ estimated by Scoville et al. (1997).

In addition to the slow evolution of the brightest sources, Rovilos et al. (2005) and Lonsdale et al. (2006) also detected some new weak $18 \mathrm{~cm}$ sources. These papers propose that these new sources are SNe, implying a mixed SN and SNR population. Supporting the SN interpretation for the new sources it was found that if their rate of appearance was taken as the $\mathrm{SN}$ rate $\nu_{\mathrm{SN}}$ and a standard IMF assumed, then the predicted SFR was consistent with that estimated from the FIR luminosity (Lonsdale et al. 2006). A possible difficulty, however, is the assumption that all corecollapse SNe give rise to RSNe luminous enough to be detected. As described in $\S 5.1 .1$ such luminous radio sources are thought to be rare. One possible explanation is that the new radio sources are instead SN/SNR transition objects (see $\S$ 5.1.1). This and other possible explanations for reconciling the rate of new radio sources and the SFR are discussed further in $\S 5.4$.

\subsubsection{Comparison of New Data and SNe Models}

The standard model for the radio emission from powerful RSNe comprises power-law emission from a synchrotron-emitting shell observed through FFA gas from the ionized CSM (Weiler et al. 2002). This absorption decreases rapidly with time as the blast wave moves outward. In $\S 4.2$ we fitted our observed source spectra around the epoch BP129 with such a power law plus FFA model and the estimated spectral indices and opacities were plotted in Figure 7. This plot shows the expected correlation between source age and opacity predicted by the RSN standard model. All the recently detected (classes $\mathrm{S}$ and $\mathrm{R}$ ) sources have high opacity, in contrast to those known to be old (class L), which have lower opacity. This strongly suggests a SN origin for the class S and R sources.

To further check whether the standard SN model applies to our sources, in Figure 9 we plot the fitted $\tau_{18}$ versus flux density at $3.6 \mathrm{~cm}$ wavelength $\left(S_{3.6}\right)$. Superimposed are loci traced by fitted models to four powerful Type II SNe with well-sampled multi- frequency light curves (Weiler et al. 2002) scaled to the distance of Arp 220. $S_{3.6}$ and $\tau_{18}$ are natural coordinates to plot our data, since for sources beyond the $3.6 \mathrm{~cm}$ peak luminosity they decouple the intrinsic strength of the synchrotron-emitting shell from the foreground absorption. In this diagram the effect of small foreground ISM opacities, which are insufficient to affect the $3.6 \mathrm{~cm}$ flux densities, is to move the $\mathrm{SN}$ tracks to the right by adding a constant $\tau_{\text {ISM. }}$.

Figure 9 shows that for all the well-fitted sources in classes $\mathrm{S}$, $\mathrm{R}$, and $\mathrm{A}$ both the opacities and luminosities are in the range defined between the most luminous detected Type IIL source SN 1979C and the Type IIn source SN 1986J. The best fit is in fact to the intermediate-luminosity Type IIn SN 1978K. However, if there is a foreground ISM opacity at $18 \mathrm{~cm}$ of $\tau_{18} \approx 1$ then SN 1986J instead provides the best fit. In both cases the required ages as of the BP129 observational epoch (2006 January) would be in the range $1-7 \mathrm{yr}$. This result suggests that sources in these three variability classes are powerful $\mathrm{SNe}$.

In contrast, it seems very difficult to explain class L sources using the standard RSN model. Three of them (W10, W17, and W42; the three relatively flat spectrum sources shown on the top row of Fig. 6) have $\tau_{18}<0.3$ and $3.6 \mathrm{~cm}$ fluxes over $250 \mu \mathrm{Jy}$. These could be consistent with objects like SN 1986J, but only if they had ages $<8 \mathrm{yr}$. However, this is less than their minimum ages $(>11 \mathrm{yr})$, ruling out a standard SN origin. Sources W18 and W39 could be SNe; however, both sources have steep spectra (see Fig. 6, middle row) and the weakness of their $3.6 \mathrm{~cm}$ flux could be ascribed to this. Finally, source W8 is in a part of the diagram that could be reached by SN models, but again the required age is much smaller than is observed.

\subsubsection{Comparison of New Data With SNR Models}

In the previous section it was shown that class $\mathrm{S}, \mathrm{R}$, and $\mathrm{A}$ sources are consistent with being RSNe. In contrast, for the older class L sources known since Smith et al. (1998) the situation is less clear. These sources have been monitored at $18 \mathrm{~cm}$ by Rovilos et al. (2005) and H. Thrall et al. (2007, in preparation) and do not show the expected flux density decay for SNe. Therefore, we should consider the possibility that these are SNRs interacting directly with the dense ISM.

To study this possibility further it is interesting to compare our class-L sources with the radio SNRs in M82, which have been well studied in a series of papers beginning with the work of Kronberg et al. (1985), Bartel et al. (1987), Ulvestad \& Antonucci (1993), and Muxlow et al. (1994). In Figure 8 we plot the $3.6 \mathrm{~cm}$ flux density versus diameter for the six well-fitted class L sources. This plot also shows the integrated fluxes of the SNRs in M82 scaled down to the distance of Arp 220. The diagonal line represents the empirical relation between surface brightness $\Sigma$ and diameter $D$ for Galactic, LMC, and M82 SNRs compiled by Huang et al. (1994) converted to luminosity and then to flux density at the distance of Arp 220. As described in $\S$ 5.1.1 SNRs in the Sedov phase of their evolution are expected to lie close to this line.

The location in the diagram of the only resolved source W42 is well above the empirical relation (see $\S 5.2 .3$ ). For the remaining sources we only have upper limits on size, as indicated by the horizontal arrows. However, if we assume that these follow the SNR luminosity-size relation, we can estimate their sizes. It is clear from the M82 data that there is at least a factor of 3 dispersion about the relation. Because we expect that our detections are biased toward the most luminous sources at a given diameter, we estimate diameters in the range $0.17-0.4 \mathrm{pc}$ with median $0.2 \mathrm{pc}$. These diameters have to be greater than $2 r_{b}$ and $2 r_{s}$ given 


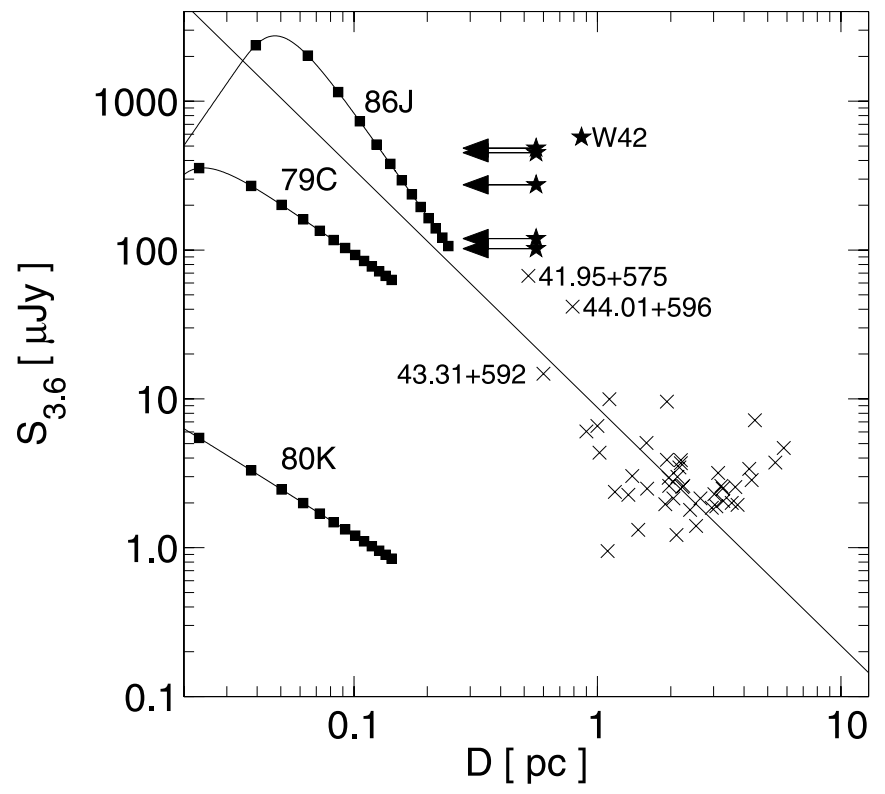

FIG. 8.- Illustration of the expected $3.6 \mathrm{~cm}$ flux density and sizes of observed SNe and SNRs if placed at the distance of Arp 220. The SN tracks for SN 1986J (Type IIn), SN 1979C ( Type IIL), and SN 1980K (Type IIL) were produced using the light-curve fits given in Weiler et al. (2002) combined with the deceleration parameter from Bietenholz et al. (2002) for SN 1986J and assuming free expansion at $10^{4} \mathrm{~km} \mathrm{~s}^{-1}$ for both SNe $1980 \mathrm{~K}$ and $1979 \mathrm{C}$. The square markers along each track indicate time evolution and are $1 \mathrm{yr}$ apart. Crosses indicate the location of 45 SNRs in M82 from Huang et al. (1994) with their fluxes scaled down to the distance of Arp 220. The diagonal line is derived from the empirical relation between SNR surface brightness and diameter $(\Sigma-D)$ fitted by Huang et al. (1994) converted to flux density at the distance of Arp 220. Stars show data for seven class L sources (W33 is not shown). Horizontal arrows indicate upper limits in size for the unresolved sources.

in equations (2) and (3), setting lower limits to the ISM pressure and density, respectively.

Taking a typical radius limit of 0.1 pc then for a Type IIn progenitor with $\dot{m}_{-4}=1$ and $v_{\mathrm{w} 1}=1$ the required ISM pressure is $P_{\mathrm{ISM}}>4 \times 10^{7} \mathrm{~K} \mathrm{~cm}^{-3}$. For more typical Type II progenitors with lower mass loss rates the pressure limit will be reduced proportionally to $\dot{m}_{-4}$ (but this may be partially offset by higher wind velocity). This pressure is similar to that found by modeling the spectral energy distribution of $\operatorname{Arp} 220$ of $10^{7} \mathrm{~K} \mathrm{~cm}^{-3}$ (Dopita et al. 2005). The somewhat higher pressure that we obtain can be explained as a selection effect in which we preferentially detect SNRs in the highest density and pressure regions (see eq. [4]).

Turning to estimates of ISM density, if in equation (2) the ejecta mass is taken as $M_{\mathrm{ej} 1}=0.5$ then $n_{\mathrm{ISM}}>3 \times 10^{4} \mathrm{~cm}^{-3}$. Although this is only a lower limit on density, we expect our detected sources to be embedded in environments with number densities close to this value. This is because given our limited sensitivity we are probably only detecting the most luminous SNRs that exist within Arp 220. Given the expected high $\nu_{\mathrm{SN}}$ of $4 \pm 2 \mathrm{yr}^{-1}$ (Lonsdale et al. 2006), these brightest and youngest SNRs in high-density environments are probably being seen only years or decades after reaching their radio maximum and hence have radii close to $r_{s}$. The estimated ISM densities for the short-wavelength detected sources are larger by a factor of a few compared to those estimated for the weaker $18 \mathrm{~cm}$ sources (see $\S 5.1 .2)$. The density estimates are consistent with the mean density estimate by Scoville et al. (1997) of $1.4 \times 10^{4} \mathrm{~cm}^{-3}$ based on $\mathrm{CO}(1-0)$ observations. Recent $\mathrm{CO}(3-2)$ observations (Narayanan et al. 2005) in ULIRGs including Arp 220 confirm, via the tight observed correlation with FIR luminosity, that most massive star formation occurs in environments with densities $>1.5 \times 10^{4} \mathrm{~cm}^{-3}$. Note that the predicted ISM densities for our bright SNR sources are just below the boundary $\left(n_{\mathrm{ISM}}>10^{5} \mathrm{~cm}^{-3}\right)$ at which they would become radiative before reaching their Sedov phase (Wheeler et al. 1980).

\subsubsection{Probable Nature of the Arp 220 Compact Sources}

Based on comparison with data from well-monitored radio $\mathrm{SN}$, there appears to be good evidence that the four class $\mathrm{R}$ sources, with rising flux density at $18 \mathrm{~cm}$ wavelength are from Type IIn RSN. Likewise, the three class S sources that are so far detected only at high frequency have spectra consistent with being even younger SNe, where the FFA is still optically thick at $18 \mathrm{~cm}$. Since, however, we have detected these sources at only one epoch we cannot yet rule out the possibility that they are steep spectrum SNe or SNRs with extreme foreground ISM absorption. Only further monitoring observations will be able to definitely decide the issue. Similar considerations apply to the three class A sources (W15, E10, and E14). The source W15 is well fitted by a standard spectral index $\alpha=-0.72$ and moderate FFA. This source shows no variability at $18 \mathrm{~cm}$ between GD17A and GD17B and is most likely a stable class L source that is just too weak to have been detected by Smith et al. (1998) and Rovilos et al. (2005). Source E14 shows inconsistency between the measured $13 \mathrm{~cm}$ flux and the two earlier $18 \mathrm{~cm}$ measurements and is hard to classify. Source E10, which shows $18 \mathrm{~cm}$ variability, is possibly an $\mathrm{SN}$, but as yet we do not have a successful spectral fit.

It should be noted that so far there is no need to resort to models of SN/SNR transition objects (see $\S$ 5.1.2) to explain the properties of class R or S sources. These transition objects, if they exist, are expected to show different multifrequency light curves, which change in unison (see $\S 5.1 .1$ ) rather than having the characteristic delay toward longer wavelengths of SN models. Future multifrequency monitoring should be able to give a conclusive result on whether any such objects exist.

For the eight detected long-lived class L sources that were in the sample of Smith et al. (1998), the analysis in $\S \S 5.1 .3$ and 5.1.4 shows that although a couple could be decades old $\mathrm{SNe}$, most have difficulties with this interpretation. The main problem, as already noted by Rovilos et al. (2005), is the high luminosity and stability of the observed light curves given their age. A striking thing about these sources is the diversity of their spectra (see Fig. 6). One source (W18) has a normal synchrotron spectral in$\operatorname{dex} \alpha=-0.62$, has a peaked spectrum caused by moderate absorption, and is similar in form to the several of the none class $\mathrm{L}$ sources shown in Figure 5. Three sources (W10, W17, and W42) have flat $(\alpha>-0.5)$ spectra, while another two sources (W8 and W39) have steep $(\alpha<-1.9)$ synchrotron spectra. Finally, W33 has a complex spectrum that cannot be fitted by standard models. For all the above class L sources (except perhaps W33) it was argued ( $\operatorname{see} \S 5.1 .4$ ) that a plausible origin was from SNRs in dense ISM environments. Still, the diversity of their spectra is a puzzle. We discuss these sources in more detail in 5.2.

\subsection{Discussion on Particular Source Classes}

In this section we discuss individual sources classes with unusual properties. All of these sources are found among our class L sources.

\subsubsection{Steep Spectrum Sources}

Two of the class L sources (W8 and W39) have fitted synchrotron power-law spectra with $\alpha<-1.2$. In addition, W7, which is not detected at any wavelength shortward of $18 \mathrm{~cm}$, must have 


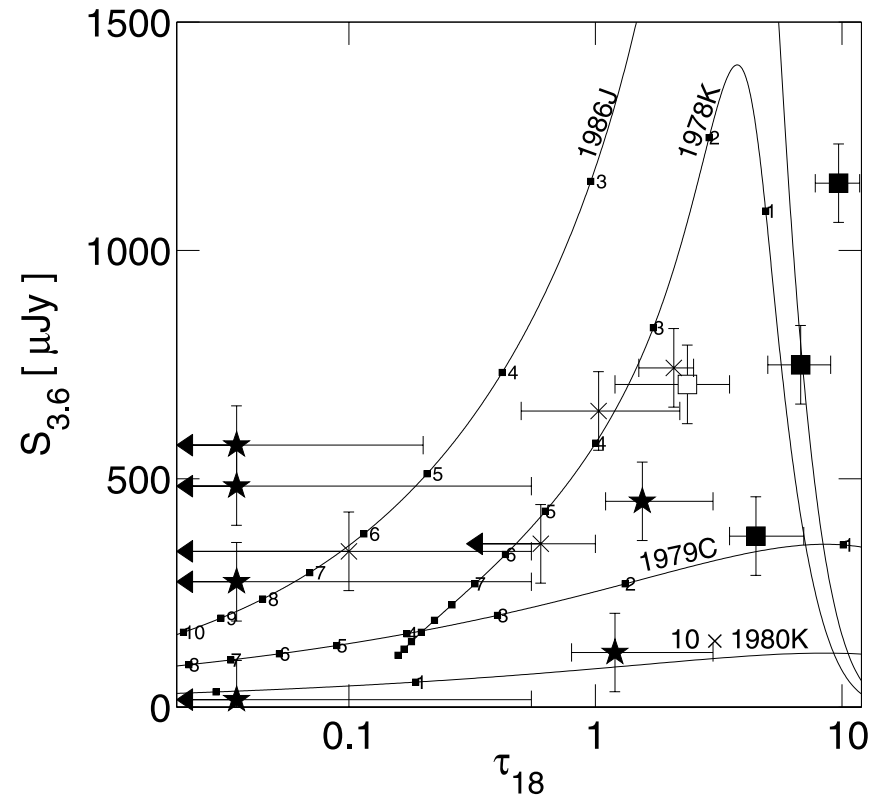

FIG. 9.-Comparison of observed flux density at $3.6 \mathrm{~cm}\left(S_{3.6}\right)$ and fitted opacity properties $\left(\tau_{18}\right)$ of the Arp 220 sources to those of well-studied RSNe. Arp 220 sources are shown using the same markers as in Fig. 7. Lines are the loci traced by the best light-curve fit (Weiler et al. 2002) to the observations of SN 1986J, SN 1978K (both Type IIn), SN 1979C, and SN 1980K (both Type IIL). Note that the curve for SN $1980 \mathrm{~K}$ has been amplified by a factor of 10 for better visualization. Markers on the tracks are labeled with the time after shock breakout in years.

$\alpha<-1.5$. These sources (see Fig. 6, middle row) are among the brightest at $18 \mathrm{~cm}$ in Lonsdale et al. (2006) and were already detected at $18 \mathrm{~cm}$ by Smith et al. (1998) in observations from 1994, implying ages of at least $11 \mathrm{yr}$. We argued in $\S 5.1 .5$ that these and other class L sources were SNRs. In our galaxy SNRs with such extreme spectral indices appear to be highly unusual. In the catalog of galactic SNRs compiled by Green ${ }^{5}$ comprising 265 objects there are none with $\alpha<-1$. However, in M82 there is one source $(42.53+61.9)$ cataloged with $\alpha=-1.84$ (McDonald et al. 2002).

Possible physical origins for the steep spectrum sources in Arp 220 are unclear. The SNR models of Berezhko \& Völk (2004) predict that in the early Sedov phase, spectral breaks that lie well above the radio-regime Adiabatic expansion caused by an SNR evolving into a low-density region (perhaps escaping its parent molecular cloud) could shift the break to lower frequencies, but only at the cost of dramatically dimming the source, which is clearly inconsistent with the fact that these sources are among the brightest observed at $18 \mathrm{~cm}$. Asvarov (2006) note that it is difficult under the standard theory of diffusive shock acceleration in SNRs to get an injected electron energy distribution giving spectral indices $\alpha<-0.6$, although a suggested possibility is that such spectra could arise if a SN exploded into a preexisting cavity with increasing density versus radius.

\subsubsection{Flat-Spectrum Low-Opacity Sources}

Three of the class L sources (W10, W17, and W42) show relatively flat $(\alpha>-0.3)$ spectra with no sign of low-frequency turnovers (see Fig. 6, top row). The fitted spectral index and the straightness of the spectra will have to be confirmed by future

\footnotetext{
5 See D. A. Green, Astrophysics Group, Cavendish Laboratory, Cambridge, UK, 2006, A Catalogue of Galactic Supernova Remnants (2006 April version), available at http://www.mrao.cam.ac.uk/surveys/snrs.
}

observations. A cautionary tale is the case of $44.01+596$ in M82, which was initially considered an AGN candidate by Wills et al. (1997) on the basis of its apparently slightly inverted spectrum between 18 and $3.6 \mathrm{~cm}$ (see Fig. 11 in Wills et al. 1997). Further observations by Allen \& Kronberg (1998) extending to shorter wavelengths showed an overall spectrum that could be fitted by a conventional $\alpha \approx-0.5$ power law plus FFA.

Despite the above caveat, the spectra for these three sources are sufficiently striking as to suggest a possible distinct class of source. In M82 a few such similar sources with $\alpha>-0.3$ exist (McDonald et al. 2002). Interestingly, these sources appear from MERLIN and VLBI imaging to show more complex spatial structures than the shell-like structures seen among the steeper spectrum sources (McDonald et al. 2002). In the starburst Arp 299 the spectra of compact sources A2, A3, and A4 (Neff et al. 2004) are also consistent with being fairly flat spectrum. Among galactic SNRs in the catalog maintained by Green, approximately $10 \%$ of the shell type remnants have $\alpha<-0.4$. There is some evidence that these flat-spectrum sources are preferentially found in dense environments; for instance the two prototypical galactic SNRs within molecular clouds, W44 (not to be confused with the source of the same name in Arp 220) and IC 443, have $\alpha=-0.39$ and $\alpha=-0.36$, respectively (Ostrowski 1999). Theoretically, spectral indices $>-0.5$ can be explained by second-order Fermi acceleration (Ostrowski 1999), among other mechanisms (Asvarov 2006).

A more exotic possibility is that these flat-spectrum objects are plerions powered by extraction of spin energy from a central neutron star. Recent VLBI observations of the archetypical radioluminous Type IIn SN 1986J (Bietenholz et al. 2004) show a compact high-frequency source that has appeared $16 \mathrm{yr}$ after the initial explosion. After the emergence of this component, the overall spectrum is observed to be flat shortward of $13 \mathrm{~cm}$. If placed at the distance of Arp 220, the observed flat-spectrum source in SN $1986 \mathrm{~J}$ would be only $74 \mu \mathrm{Jy}$ in flux density; however, the central source may still be rising in luminosity. Theoretically (Bietenholz et al. 2004), the extraction of energy from a central pulsar could produce a source up to 5 times as luminous as presently observed in SN 1986J and hence be comparable in luminosity to the flat-spectrum sources we see in Arp 220. Finally, there is the possibility, which we mention for completeness, that one or more of the flat-spectrum sources might be due to an AGN or an accreting intermediate-mass black hole (Wrobel \& Ho 2006).

\subsubsection{W42: Resolved Flat-Spectrum Source}

W42 is the only source showing evidence for resolution at $3.6 \mathrm{~cm}$. A deconvolved FWHM of 2.3 mas is obtained using a Gaussian fit that corresponds to a linear size of $0.86 \mathrm{pc}$. Although we cannot rule out the possibility that it could be two or more sources close together, we here assume a single source. W42 is one of the three sources showing relatively flat spectra (see previous section). The source was detected in the original discovery observations of Smith et al. (1998), and hence its minimum age is $11 \mathrm{yr}$, implying an upper limit of $40,000 \mathrm{~km} \mathrm{~s}^{-1}$ for its mean expansion velocity. However, monitoring at $18 \mathrm{~cm}$ (see Fig. 3 in Rovilos et al. 2005) shows a stable light curve. This stability persists to the latest $18 \mathrm{~cm}$ measurements (see Table 2 of $\mathrm{H}$. Thrall et al. 2007 , in preparation) and suggests that the source is significantly older.

Estimates of minimum energy in relativistic particles and fields with W42 can be made using the standard equipartition argument (see Longair 1994). Recently, problems with this standard derivation have been pointed out by Beck \& Krause (2005), who provide revised formulae to calculate minimum fields and energies for cases with $\alpha<-0.5$. Since for spectral indices close to 
-0.5 differences to the classical result are relatively small, we instead use the version of the classical formula from Beck \& Krause (2005), which is applicable for any $\alpha$, to calculate the minimum energy requirement for W42.

Along the position angle corresponding to the beam minor axis, the FWHM of W42 is about twice as big as the beam FWHM (see Fig. 4, left), which is consistent with a thin shell of diameter 2.3 mas or $0.86 \mathrm{pc}$. If we assume that this radio-emitting shell has a ratio of inner to outer radius of 0.8 , similar to the SNR shells imaged in M82 (see for instance Kronberg et al. 1985; Ulvestad \& Antonucci 1993; Muxlow et al. 1994), then the volume filling factor $\phi=0.51$. We take the upper limit of integration of the spectrum to be $8.6 \mathrm{GHz}$, which is the highest frequency for which we so far detect emission. A critical parameter in the classical formula is the ratio of total energies in protons to electrons $K$. For a source with a flat spectral index this ratio will be similar to the relative number densities of protons and electrons at fixed energy, which in $\mathrm{SNe} / \mathrm{SNR}$ is probably in the range 40-100 (see Beck \& Krause 2005); we adopt $K=100$. Using the above parameters the resulting minimum total energy in W42 is $\sim 2 \times 10^{50} \mathrm{ergs}$. This value is a weak function of the assumed $\alpha$ and upper frequency cutoff. The energy scales as $(1+K)^{4 / 7} \phi^{3 / 7}$, so the energy requirement could be decreased if we are seeing radio emission from low volume filling factor filaments or if the overall structure is not spherical but bipolar or elongated.

Huang et al. (1994) estimate that for young SNRs entering the Sedov phase, in which a large fraction of the initial kinetic energy has been converted to thermal energy, the sum of energy in relativistic particles and fields is $2 \%$ of this thermal energy. If W42 has the same energy conversion efficiency, then the total kinetic energy released by its progenitor $\mathrm{SN}$ is $10^{52} \mathrm{ergs}$, which is 10 times larger than the canonical value of $10^{51}$ ergs expected for core-collapse SNe. There is, however, increasing evidence that hypernova remnants created from such energetic explosions do occur (see, for instance, Urošević et al. 2005). Longperiod gamma-ray bursts are now thought to come from a subclass of Type Ibc SN (Woosley \& Bloom 2006) with total kinetic energies $\sim 5 \times 10^{51}$ ergs. Such an energetic hypernova origin for W42 would be consistent with its high luminosity relative to the SNR luminosity- $D$ relation (see Fig. 8), which, according to Berezhko \& Völk (2004), depends only on its total kinetic energy.

It is interesting to compare W42 with the two brightest $3.6 \mathrm{~cm}$ sources in M82 (see Fig. 8), both of which have similar diameters but luminosities 10 times smaller. The source $41.95+575$ is the brightest, most compact source in M82 and appears as a bipolar structure of $\sim 0.5 \mathrm{pc}$ along its major axis (Beswick et al. 2006). Like W42, it also lies well above the luminosity- $D$ relation, but it is less extreme in this regard. It also has some other differences, including a significant decrease in flux density of $7.1 \% \mathrm{yr}^{-1}$ (Beswick et al. 2006), an estimated expansion velocity of only $\sim 1500-2000 \mathrm{~km} \mathrm{~s}^{-1}$ and a conventional peaked radio spectrum. The next most luminous source in M82 is 44.01+596, which shows a shell structure of diameter 0.79 pc (Huang et al. 1994; McDonald et al. 2001). This is probably the closest analog to W42; as noted in the first paragraph of this section, this object was initially thought to have an unusually flat spectrum, but subsequent observations over a wider frequency range showed a conventional peaked spectrum source.

\subsubsection{Complex Spectrum Source W33}

The region around source W33 has a complex structure as shown by Figure 4 (right). Figure 6 shows the spectrum evaluated at the position of W33 itself, as marked by a cross in Figure 4 (right). Note in this particular spectrum the vertical axis is the brightness in $\mu \mathrm{Jy}_{\text {beam }}{ }^{-1}$. Because of its complex nature, a power law plus FFA model did not give a good fit to this spectrum (see $\S 4.3)$. Another unusual property of W33 is that at $6 \mathrm{~cm}$ the source has shown a rapid decrease in flux density of $40 \%$ in just over $2 \mathrm{yr}$ (the time difference between the BN022 and BP129 epochs). Being a class $\mathrm{L}$ source, it is known to have a long lifetime. The light curve at $18 \mathrm{~cm}$ shows no overall trend but does have significant variability between epochs (Rovilos et al. 2005).

The $13 \mathrm{~cm}$ image in Figure 4 shows evidence for a possible extension to the north of W33, which appears to be significant with respect to the noise. However, no such extension is seen in the sensitive $18 \mathrm{~cm}$ image made from data taken less than a year before (GD17B). This fact plus similar weaker extensions to the source immediately to the west (W34) suggests that the W33 extension may simply be an imaging artifact. We cannot, however, exclude the possibility that it could be due to an adjacent Type Ib/c SN, which is expected to evolve on timescales of $<100$ days but can in some cases reach or exceed the luminosity of Type IIn SNs (see Chevalier et al. 2006). It is interesting that the region around W33 lies close to one end of a patch of high brightness $18 \mathrm{~cm}$ emission (H. Thrall et al. 2007, in preparation), which may be either high-brightness diffuse emission or confused emission from many compact sources. The evidence seems to suggest that there may be intense highly concentrated star formation activity in this region.

\subsection{Foreground ISM Free-Free Opacities}

The results of the spectral fitting in $\S 4$ give overall estimated free-free opacities for each source which are the sum of a foreground ISM opacity and a local opacity due to an ionized CSM. Despite this they can be used to constrain properties of the ionized ISM because the fitted $\tau_{18}$ gives a firm upper limit on the ionized ISM opacity at $18 \mathrm{~cm} \tau_{\text {ISM,18 }}$ along each line of sight. Furthermore, it was argued in $\S 5.1 .3$ that sources of classes R, S and A are probably RSNe with significant CSM FFA, while class L sources were most likely SNRs with no CSM absorption. If we accept these conclusions, then the measured opacities for class L sources are not just upper limits to $\tau_{\mathrm{ISM}, 18}$ but actual measurements. Among the six type L sources with good fits, four were found to have $1 \sigma$ upper limits on opacity of 0.3 . For these lines of sight (taking into account also possible systematic calibration errors) we estimate that $\tau_{\text {ISM, } 18}<0.6$ (see Fig. 7). The other two sources had $\tau_{18}=1.2$ and 1.5 , respectively. Although we only have small number statistics, these results are suggestive that in the western nucleus of Arp 220 there is a patchy FFA ISM with a median opacity of less than 1 . It is presently impossible to give any estimate for the eastern nucleus, because of the lack of good spectral fits to any L-type source.

It is interesting to compare our results with those of Anantharamaiah et al. (2000), who modeled the ionized thermal gas component in Arp 220 by jointly fitting to radio recombination lines and the continuum spectrum. The predictions of this model have recently been confirmed by VLA observations of another recombination line (Rodríguez-Rico et al. 2005). Anantharamaiah et al. (2000) present an integrated continuum spectrum (encompassing both nuclei but dominated at most frequencies by the western nucleus). This spectrum shows a steep spectrum non thermal power law from 3 to $30 \mathrm{GHz}$. From $300 \mathrm{MHz}$ to $2 \mathrm{GHz}$ there is flat-spectrum region, and below $300 \mathrm{MHz}$ a sharp cutoff. Anantharamaiah et al. (2000) invoke a nonthermal synchrotron continuum plus three ionized gas components (A1, A2, and D) to jointly explain this spectrum and observed radio recombination lines. The A2 component (from high-pressure $\mathrm{H}$ II regions) has a very low covering factor and in continuum affects only high 
frequencies. The A1 and D components have $\tau_{\text {ISM }, 18}=0.97$ and 0.02 with area covering factors of 0.7 and 1 , respectively. These two components explain the flat region below $2 \mathrm{GHz}$ (where $\mathrm{A} 1$ starts to become optically thick) and the sharp drop below $300 \mathrm{MHz}$ (where D becomes optically thick). The estimated opacities derived for our compact L source seem broadly to be consistent with this model, with some sources having little foreground FFA and others a moderate amount.

\subsection{Star Formation Rates and the Nature of Arp 220's Starburst}

As noted by Lonsdale et al. (2006), the SFR inferred from the FIR luminosity is consistent with the rate of appearance of new radio sources $\left(4 \pm 2 \mathrm{yr}^{-1}\right)$ if all or most core-collapse $\mathrm{SNe}$ give rise to a detectable radio source. However, there is evidence from their luminosity, radio spectra, and evolutionary timescale confirming that these new sources are Type IIn SNe. This class of $\mathrm{SNe}$ are thought to be relatively rare in normal galaxies (Cappellaro et al. 1997), comprising only $2 \%$ of core-collapse $\mathrm{SNe}$ (although due to their extreme luminosities, they are much more common in compilations of detected SNe). In addition, Avishay Gal-Yam et al. (2007) have recently identified the progenitor of the Type IIn SN $2005 \mathrm{gl}$ as a luminous blue variable (LBV) star. Such stars are known to have initial masses in excess of $\sim 80 M_{\odot}$. If a standard IMF is assumed, then the fraction of core-collapse SNe with progenitors in this mass range is $\sim 3 \%$, similar to the expected fraction of Type II SNe, implying that all or most Type IIn progenitors are LBVs. If this is true, then under conventional RSNe and starburst models the observed rate of appearance of new compact radio sources could be up to 50 times larger than the number predicted from the SFR. A full discussion of this issue will be left to a future paper, but it is interesting to speculate on possible resolutions of this apparent inconsistency.

For instance, the density in the nuclear region of Arp 220 could be so high that the adopted paradigm of an early SN phase followed by an SNR phase does not apply. Instead, every corecollapse SN interacts directly with the dense ISM and gives a bright radio source. Hence the observed rate of new radio sources matches the prediction from the SFR (Lonsdale et al. 2006). However, so far it seems that the properties of the new sources are consistent with standard RSNe interacting with their stellar winds. In this case a possible explanation is that the IMF in Arp 220 has an unusual overabundance of very massive stars. Such a scenario for Arp 220 would be very interesting since it would suggest a difference in star formation mechanisms compared to those found in galactic disks. Nevertheless, a recent review (Elmegreen 2005) points out that there are considerable uncertainties in measuring the IMF in massive star clusters. Therefore, there is no decisive evidence in favor of a top-heavy IMF in starburst galaxies.

Another possible scenario consists of a normal IMF but that the late stages in the evolution of massive stars is fundamentally different in Arp 220 due to the very high (stellar) density ULIRG environment. It has been predicted that binary systems will give more Type IIb/IIL sources at the expense of radio-weak Type IIP (Nomoto et al. 1996; Chevalier 2006). Possibly some mechanism might exist such that a higher fraction of multiple star systems or close stellar encounters also dramatically increases the fraction of the extremely luminous Type IIn events.

One last possibility is that the SFR of the starburst is highly variable on the timescale of massive star lifetimes, and we are now observing Arp 220 just when the massive stars formed in a previous burst are exploding as $\mathrm{SNe}$. Based on their observations of radio recombination lines, one of the scenarios discussed in Anantharamaiah et al. (2000) is that the star formation in Arp 220 could be occurring in intense but very short bursts, each lasting a few times $10^{5} \mathrm{yr}$ with a SFR in the order of $10^{3} M_{\odot} \mathrm{yr}^{-1}$, separated by a few times $10^{6} \mathrm{yr}$.

To check whether this scenario can provide enough massive stars to explain the observed luminous RSN rate, consider that the previous burst lasted $3 \times 10^{5} \mathrm{yr}$ with a SFR of 10 times the averaged SFR estimated by Anantharamaiah et al. (2000), i.e., $2400 M_{\odot} \mathrm{yr}^{-1}$, thus giving a total mass of $7 \times 10^{8} M_{\odot}$ in new stars. If a Salpeter IMF with power-law exponent of -2.3 and a low-mass cutoff of $0.5 M_{\odot}$ is assumed (Kroupa 2002), then $4.5 \times 10^{5}$ of these stars are estimated to be more massive than $80 M_{\odot}$ (note that no upper mass limit was used; if in contrast, an upper limit of $120 M_{\odot}$ is assumed, this estimate is reduced by a factor of 2$)$. If the previous burst occurred one LBV lifetime ago $\left(3 \times 10^{6} \mathrm{yr}\right.$; Massey et al. 2001), these massive stars would be exploding over a period defined by the sum of the burst length and the stellar lifetimes. For such massive stars, the H-burning time is known to be a weak function of mass (Massey et al. 2001); therefore, assuming a dispersion in their lifetimes of $10 \%$ (which is comparable to the burst duration), all these massive stars would explode over a period of $6 \times 10^{5} \mathrm{yr}$, implying a luminous RSN rate of $0.75 \mathrm{yr}^{-1}$, which is of the order of the observed rate.

The above calculation shows that despite the many uncertainties the short burst explanation for the large rate of powerful $\mathrm{RSNe}$ is feasible. However, a possible problem is that it requires that fortuitously there was a burst exactly $3 \times 10^{6}$ yr ago to provide the RSNe we see plus another ongoing burst to provide the large number of very young $\left(<10^{5} \mathrm{yr}\right)$ overpressured $\mathrm{H}$ iI regions required by Anantharamaiah et al. (2000); moreover, this would have to be true for both nuclei. Scoville et al. (1997) argue that the two nuclei are embedded within a larger scale disk and are mutually orbiting each other. We can speculate that one way to obtain the needed star formation history would be if their orbit were at least somewhat elliptical and bursts were triggered by their periodic periapsis passage. Based on the separation of the nuclei and their relative systemic velocities, Scoville et al. (1997) argue for an orbital period for the double nucleus of $\sim 4.6 \times 10^{6} \mathrm{yr}$. Although this period estimate is uncertain encouragingly it is comparable to LBV lifetimes.

\section{CONCLUSIONS AND FUTURE PROSPECTS}

The main conclusions of this paper are as follows:

1. For the first time we have detected the compact radio sources in Arp 220 at wavelengths shorter than $18 \mathrm{~cm}$ (see $\S 2.2$ ). Previous failures to detect the compact sources at $6 \mathrm{~cm}$ appear to be largely due to using a phase calibrator that was too distant, possibly combined with the observations being made near the maximum of the solar cycle. We also uncovered an astrometric error of $\sim 100$ mas in declination in previously published absolute $\mathrm{OH}$ maser and $18 \mathrm{~cm}$ continuum VLBI positions (see $\S 2.4$ ).

2. A total of 18 sources (all but three in the western nucleus) are clearly detected at short wavelengths (see Table 2). For these we construct estimated radio spectra from our simultaneous measurements at $3.6,6,13 \mathrm{~cm}$ and a nonsimultaneous $18 \mathrm{~cm}$ measurement. A wide variety of spectral shapes are seen ranging from inverted through peaked to steep (see $\S 3.4$ ). Most can be well fitted by a simple model consisting of a synchrotron power law plus foreground free-free absorption (see $\S 2.4$ ).

3 . There is evidence that younger sources have higher freefree absorption consistent with what is expected from RSN models (see $\S 5.1 .3$ ). In particular, four sources than have recently appeared at $18 \mathrm{~cm}$ seem well fitted by such models. Newly cataloged sources seen only at short wavelength may be even 
younger SNe or old SNRs with especially large foreground ISM opacity. In general, for sources that were not detected in the original discovery observations of Smith et al. (1998), the combination of their fitted free-free opacities, luminosities, and age limits is consistent with what is expected for Type IIn RSNe.

4. The bright $18 \mathrm{~cm}$ sources originally detected by Smith et al. (1998) in addition to showing little time variability also have lower fitted free-free opacities than the other sources. The underlying synchrotron spectral index of these sources shows a wide range and contains both relatively flat $\alpha>-0.3$ and steep spectrum $(\alpha<-1)$ sources (see $\S 5.1 .5)$. It seems difficult to interpret these sources as standard RSNe because of their combined age and luminosity (see $\S 5.1 .3$ ). Models in which they are instead young SNRs interacting with the surrounding ISM are more successful. If they follow the same luminosity-size relation as the SNRs in M82, their luminosities imply diameters of $0.2 \mathrm{pc}$ and surrounding ISM of densities in the range $10^{4}<n_{\text {ISM }}<10^{5} \mathrm{~cm}^{-3}$. Ages for these sources would be only $30-50 \mathrm{yr}$, similar to those estimated by Lonsdale et al. (2006).

5 . One source (W42) is probably resolved at $3.6 \mathrm{~cm}$ (diameter $=$ $0.86 \mathrm{pc}$ ). Estimates of the minimum total energy in particles and fields (see $\S 5.2 .3$ ) suggest that the initial kinetic energy of the supernovae was larger than in typical core-collapse SN and could be as high as $10^{52}$ ergs.

6. Source W33 shows a complex spectrum and has evidence for significant epoch-to-epoch variability (see $\S 5.2 .4)$. It is located in a crowded region close to a patch of apparently high brightness extended $18 \mathrm{~cm}$ emission. There is possible evidence of extended structure at $13 \mathrm{~cm}$, but this may be caused by imaging artifacts. The area around W33 has a high density of compact sources and is a highly active star formation region.

7. Based on the fitted free-free absorption toward the stable SNR candidates in the western nucleus we are able constrain the opacity due to the ionized component of the ISM. Four sources have opacities at $18 \mathrm{~cm}$ of $\tau_{18 \text {,ISM }}<0.6$, and the other two have $\tau_{18 \text {,ISM }}=1.2$ and 1.5 , respectively. These results are in good agreement with the model of Anantharamaiah et al. (2000), which postulates two phases of ionized gas affecting absorption at low frequency, one with covering factor 0.7 and opacity $\tau_{18 \text {,ISM }}=1$, and the other with a covering factor of 1 and $\tau_{18, \mathrm{ISM}}=0.02$.

8. Lonsdale et al. (2006) found that the rate of appearance of new radio sources approximately equals that expected from the FIR derived SFR if every core-collapse SN gives rise to a bright radio source. However, slowly evolving SN sources as bright as those detected in Arp 220 belong to the Type IIn class which are thought to be relatively rare in galactic disks. Consistent with this is recent identification of Type IIn progenitors as extremely massive LBV stars $\left(>80 M_{\odot}\right)$. In $\S 5.4$ we discuss possible explanations to this apparent conflict. Among the possibilities are that a totally different RSN paradigm applies in ULIRGs, that a topheavy stellar IMF, or that nonstandard stellar evolution applies. A final possibility is that the recent starburst activity occurred in a very short but intense burst that we are now observing just as the most massive stars explode as $\mathrm{SNe}$.

9. In $\oint 5.2 .2$ we noted the outside possibility that one of the flatter spectrum sources could be an AGN; likewise, the source with an unusual spectrum, W33 (see $\S$ 5.2.4), might be another possible candidate. However, based on present data there is no convincing case of any source that requires such an AGN explanation.

The detection of the Arp 220 compact sources at short wavelengths opens up many interesting future observational possibilities. The observations presented here only scratch the surface. Recently conducted (2006 May) and proposed global VLBI observations using the world's largest telescopes and largest bandwidths will give images 10 times more sensitive than those presented in this paper. These data will help determine the spectral properties of the weaker sources and constrain systematic differences with luminosity. With a larger number of detected sources we can also test for possible differences in the spatial distribution of sources with different spectra. Observations at the shortest wavelengths can be used to see if our apparently flat spectrum remain so to the highest frequencies. In addition, because SNe evolve much faster at short wavelengths, source monitoring will produce much faster results than at $18 \mathrm{~cm}$. Multiwavelength light-curve monitoring will allow us to make detailed SN fits for stellar mass loss properties (indirectly probing stellar evolution). In addition, such fitting will give more accurate estimates of foreground FFA allowing us to map the spatial distribution of the ionized ISM.

Finally, future short-wavelength observations may be able to resolve or set useful limits on source sizes. One candidate SNR source (W42) has probably already been resolved. A SN in free expansion at $10^{4} \mathrm{~km} \mathrm{~s}^{-1}$ will after $5 \mathrm{yr}$ have diameter of $0.1 \mathrm{pc}$ (0.3 mas), which is within the current observational resolving capabilities. Likewise, we can check the prediction that those sources we have identified as SNRs have diameters of $0.2 \mathrm{pc}$ (0.6 mas). Finally, if such SNRs follow the expected luminosity- $D$ correlation then the weaker sources are expected to be larger, a prediction that can be checked by future more sensitive observations.

R. P. acknowledges a Chalmers University Ph.D. student stipend, and J. C. acknowledges a Swedish VR grant. This work was partially supported by NSF grant AST 03-52953 to Haystack Observatory. The European VLBI Network is a joint facility of European, Chinese, South African, and other radio astronomy institutes funded by their national research councils. The Arecibo Observatory is the principal facility of the National Astronomy and Ionosphere Center, which is operated by the Cornell University under a cooperative agreement with the National Science Foundation. The National Radio Astronomy Observatory is a facility of the National Science Foundation operated under cooperative agreement by Associated Universities, Inc. We thank the anonymous referee for his/her valuable comments and suggestions.

\section{REFERENCES}

Alberdi, A., Colina, L., Torrelles, J. M., Panagia, N., Wilson, A. S., \& Garrington, S. T. 2006, ApJ, 638, 938

Allen, M. L., \& Kronberg, P. P. 1998, ApJ, 502, 218

Anantharamaiah, K. R., Viallefond, F., Mohan, N. R., Goss, W. M., \& Zhao, J. H. 2000, ApJ, 537, 613

Arthur, S. J. 2006, preprint (astro-ph/0605533)

Asvarov, A. I. 2006, A\&A, 459, 519

Bartel, N., et al. 2002, ApJ, 581, 404 1987, ApJ, 323, 505

Beasley, A. J., \& Conway, J. E. 1995, in ASP Conf. Ser. 82, Very Long Baseline Interferometry and the VLBA, ed. J. A. Zensus, P. J. Diamond, \& P. J. Napier (San Francisco: ASP), 328
Beck, R., \& Krause, M. 2005, Astron. Nachr., 326, 414

Berezhko, E. G., \& Völk, H. J. 2004, A\&A, 427, 525

Beswick, R. J., et al. 2006, MNRAS, 369, 1221

Bietenholz, M. F., Bartel, N., \& Rupen, M. P. 2002, ApJ, 581, 1132 2004, Science, 304, 1947

Cappellaro, E., Turatto, M., Tsvetkov, D. Y., Bartunov, O. S., Pollas, C., Evans, R., \& Hamuy, M. 1997, A\&A, 322, 431

Chevalier, R. 2006, preprint (astro-ph/0607422)

Chevalier, R. A. 1982a, ApJ, 259, L85

1982b, ApJ, 259, 302

1998, ApJ, 499, 810

2005, ApJ, 619, 839 
Chevalier, R. A., \& Fransson, C. 1994, ApJ, 420, 268 2001, ApJ, 558, L27

Chevalier, R. A., Fransson, C., \& Nymark, T. K. 2006, ApJ, 641, 1029

Chevalier, R. A., Li, Z.-Y., \& Fransson, C. 2004, ApJ, 606, 369

Corbett, E. A., et al. 2002, ApJ, 564, 650 2003, ApJ, 583, 670

Cowsik, R., \& Sarkar, S. 1984, MNRAS, 207, 745

Dopita, M. A., et al. 2005, ApJ, 619, 755

Draine, B. T., \& Woods, D. T. 1991, ApJ, 383, 621

Elmegreen, B. G. 2005, in Starbursts, From 30 Doradus to Lyman Break Galaxies, ed. R. de Grijs \& R. M. González Delgado (Dordrecht: Springer), 57

Gal-Yam, A., et al. 2007, ApJ, 656, 372

Huang, Z. P., Thuan, T. X., Chevalier, R. A., Condon, J. J., \& Yin, Q. F. 1994, ApJ, 424, 114

Kronberg, P. P., Biermann, P., \& Schwab, F. R. 1985, ApJ, 291, 693

Kroupa, P. 2002, Science, 295, 82

Longair, M. S. 1994, High Energy Astrophysics, Vol. 2. Stars, the Galaxy, and the Interstellar Medium (2nd ed.; Cambridge: Cambridge Univ)

Lonsdale, C. J., Diamond, P. J., Thrall, H., Smith, H. E., \& Lonsdale, C. J. 2006, ApJ, 647, 185

Massey, P., DeGioia-Eastwood, K., \& Waterhouse, E. 2001, AJ, 121, 1050

McDonald, A. R., Muxlow, T. W. B., Pedlar, A., Garrett, M. A., Wills, K. A., Garrington, S. T., Diamond, P. J., \& Wilkinson, P. N. 2001, MNRAS, 322, 100

McDonald, A. R., Muxlow, T. W. B., Wills, K. A., Pedlar, A., \& Beswick, R. J. 2002, MNRAS, 334, 912

Muxlow, T. W. B., Pedlar, A., Wilkinson, P. N., Axon, D. J., Sanders, E. M., \& de Bruyn, A. G. 1994, MNRAS, 266, 455

Narayanan, D., Groppi, C. E., Kulesa, C. A., \& Walker, C. K. 2005, ApJ, 630, 269

Natta, A., \& Panagia, N. 1984, ApJ, 287, 228

Neff, S. G., Ulvestad, J. S., \& Teng, S. H. 2004, ApJ, 611, 186

Nomoto, K., Iwamoto, K., Suzuki, T., Pols, O. R., Yamaoka, H., Hashimoto, M., Hoflich, P., \& van den Heuvel, E. P. J. 1996, in IAU Symp. 165, Compact Stars in Binaries, ed. J. van Paradijs, E. P. J. van den Heuvel, \& E. Kuulkers, 119
Ostrowski, M. 1999, A\&A, 345, 256

Parra, R., Conway, J., Appleton, P., \& Pihlström, Y. 2005, in AIP Conf. Proc. 783, The Evolution of Starbursts, ed. S Hüttemeister, E. Manthey, \& K. Weis (Melville: AIP), 241

Petrov, L., Kovalev, Y. Y., Fomalont, E., \& Gordon, D. 2005, AJ, 129, 1163

Pradel, N., Charlot, P., \& Lestrade, J.-F. 2005, in ASP Conf. Ser. 340, Future Directions in High-Resolution Astronomy, ed. J. Romney \& M. Reid (San Francisco: ASP), 538

Rodríguez-Rico, C. A., Goss, W. M., Viallefond, F., Zhao, J.-H., Gómez, Y., \& Anantharamaiah, K. R. 2005, ApJ, 633, 198

Rovilos, E., Diamond, P. J., Lonsdale, C. J., Lonsdale, C. J., \& Smith, H. E. 2003, MNRAS, 342, 373

Rovilos, E., Diamond, P. J., Lonsdale, C. J., Smith, H. E., \& Lonsdale, C. J. 2005, MNRAS, 359, 827

Scoville, N. Z., Yun, M. S., \& Bryant, P. M. 1997, ApJ, 484, 702

Smith, H. E., Lonsdale, C. J., Lonsdale, C. J., \& Diamond, P. J. 1998, ApJ, 493, L17

Soderberg, A. M., Kulkarni, S. R., Berger, E., Chevalier, R. A., Frail, D. A., Fox, D. B., \& Walker, R. C. 2005, ApJ, 621, 908

Thompson, A. R., Moran, J. M., \& Swenson, G. W., Jr. 2001, Interferometry and Synthesis in Radio Astronomy (2nd ed.; New York)

Ulvestad, J. S., \& Antonucci, R. R. J. 1993, in BAAS, 25, 842

Urošević, D., Pannuti, T. G., Duric, N., \& Theodorou, A. 2005, A\&A, 435, 437

van Marle, A. J., Langer, N., \& García-Segura, G. 2004, Rev, Mex. AA Conf. Ser. 22, Gravitational Collapse: From Massive Stars to Planets, ed. G. GarciaSegura, G. Tenorio-Tagle, J. Franco, \& H. W. Yorke, 136

Weiler, K. W., Panagia, N., Montes, M. J., \& Sramek, R. A. 2002, ARA\&A, 40, 387

Wheeler, J. C., Mazurek, T. J., \& Sivaramakrishnan, A. 1980, ApJ, 237, 781

Wills, K. A., Pedlar, A., Muxlow, T. W. B., \& Wilkinson, P. N. 1997, MNRAS, 291, 517

Woosley, S. E., \& Bloom, J. S. 2006, ARA\&A, 44, 507

Wrobel, J. M., \& Ho, L. C. 2006, ApJ, 646, L95

Wrobel, J. M., Walker, R. C., \& Benson, J. M. 2000, VLBA Correlator Memo 24 